\title{
Heat Transfer Augmentation in Unsteady Conjugate Thermal Systems - Part II: Applications
}

\author{
Richard Mathie $^{\mathrm{a}}$, Hajime Nakamura ${ }^{\mathrm{b}}$, Christos N. Markides ${ }^{\mathrm{a}, *}$ \\ ${ }^{a}$ Department of Chemical Engineering, Imperial College London, \\ South Kensington Campus, London SW7 2AZ, U.K. \\ ${ }^{b}$ Department of Mechanical Engineering, National Defence Academy of Japan, \\ 1-10-20 Hashirimizu, Yokosuka 239-8686, Japan.
}

\begin{abstract}
Many energy conversion and other thermal-fluid systems exhibit unsteady convective heat exchange. In such systems, generic spatiotemporal variations in the flow give rise to variations in the heat flux for a given fluid-solid temperature difference, which can be interpreted as spatiotemporal fluctuations of the instantaneous heat transfer coefficient. These variations can lead to unsteady conjugate heat transfer, in which the exchanged heat flux arises from an interaction between the bulk fluid temperature and the temperature in the solid. Further, the nonlinear coupling between the fluctuating temperature differences and the heat transfer coefficient can lead to an effect we refer to as augmentation, which quantitatively describes the ability of a particular arrangement to have a different time-mean heat flux from the product between the mean heat transfer coefficient and the mean temperature difference across the fluid. It is important to be able to understand and to model in a simple framework the effects of the material properties, the geometry and the character of the heat transfer coefficient on the thermal response of the fluid-solid system, and consequently to predict the overall heat transfer performance of these systems.

This paper, which follows on from its predecessor (Mathie and Markides, 2012a), is concerned with the phenomenon of augmentation in simple, one-dimensional, unsteady and conjugate fluid-solid systems. A simple semi-analytical one-dimensional model of heat transfer with a time-varying heat transfer coefficient, which was presented in Mathie and Markides (2012a), is applied herein to two different paradigm problems. Such a model can be an important tool in the design of improved heat exchangers and thermal insulation, through for example, the novel selection of materials to exploit these augmentation effects. The first flow considered is a thin, wavy fluid film flowing over a heated plate. This film flow exhibits a periodic fluctuation in the heat transfer coefficient, that is linked to the wavy interfacial deformations of free surface of the liquid film. The second flow considered concerns the heat transfer behind a backwards-facing step, which exhibits broadband fluctuations in the heat transfer coefficient due to the flow separation and turbulence behind
\end{abstract}

\footnotetext{
*Corresponding author. Tel.: +44 (0)20 75941601.

Email address: c.markides@imperial.ac.uk (Christos N. Markides)
} 
the step. The model predictions of the augmentation ratio for these problems are also compared to direct measurements from each case. Good agreement is observed with the experimental results for the global heat transfer trends. In both cases the augmentation ratio was negative, reflecting a reduction in time-averaged heat transfer. For the backwards-facing step flow a low magnitude of augmentation ratio was observed, however, the thin film flows exhibited augmentation ratios of as high as $10 \%$. 


\section{Nomenclature}

\section{Material Properties}

$\alpha$ Thermal diffusivity, $\frac{k}{\rho c}$

$\epsilon$ Thermal effusivity, $\sqrt{k \rho c}$

$\rho$ Density

$\nu$ Kinematic viscosity

$C^{*}$ Volumetric (or volume-specific) heat capacity ratio, $\frac{\rho_{\mathrm{f}} c_{\mathrm{f}}}{\rho_{\mathrm{s}} c_{\mathrm{s}}}$

c Specific heat capacity

$k$ Thermal conductivity

\section{Variables}

Bi Biot number

$N u$ Nusselt number

$\operatorname{Pr}$ Prandtl number

Re Reynolds number

$\delta$ Thermal diffusion length, $\sqrt{\frac{2 \alpha}{\omega}}=\sqrt{\frac{2 k}{\rho c \omega}}$

$\Gamma$ Fluid flow-rate per unit channel width $w, \frac{Q}{w}$

$\sigma$ Standard deviation of subscripted variable

$\omega$ Oscillation (angular) frequency

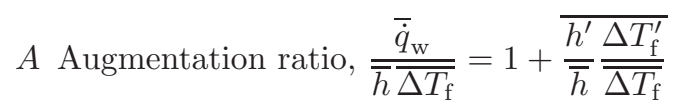

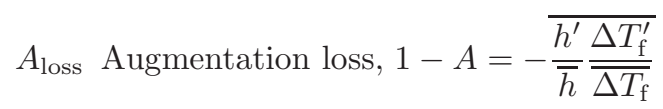

a Solid thickness

$a^{*}$ Normalised solid thickness, $\frac{a}{\delta_{\mathrm{s}}}$

$d$ Fluid domain thickness

$d^{*}$ Normalised fluid thickness, $\frac{d}{a}$ 
$g$ Gravitational acceleration

$C_{\mathrm{R}} \quad$ Heat capacity ratio, $C^{*} d^{*}=\frac{\rho_{\mathrm{f}} c_{\mathrm{f}}}{\rho_{\mathrm{s}} c_{\mathrm{S}}} \frac{d}{a}$

$h$ Heat transfer coefficient

$L_{\text {th }}$ Thermal development length

$Q$ Volumetric flow-rate

$\dot{q}$ Heat flux

$T$ Temperature field

$\Delta T_{\mathrm{f}}$ Fluid temperature difference, $T_{\mathrm{f}, \mathrm{b}}-T_{\mathrm{w}}$ or $T_{\mathrm{f}, \infty}-T_{\mathrm{w}}$

$t$ Time

$U$ Fluid velocity field

$U^{*}$ Normalised fluid velocity, $\frac{\nu_{\mathrm{f}}}{\omega d^{2}} R e$

$w$ Width of flow perpendicular to the flow direction

$x$ Coordinate in the streamwise direction

$y$ Coordinate normal to the wall and starting from the outer (unwetted) solid surface, $\widetilde{y}+a$

$\widetilde{y}$ Coordinate normal to the wall and starting from the inner (wetted) solid surface, $y-a$

$Z$ Thermal impedance of the solid in the frequency domain

$Z^{*}$ Normalised thermal impedance of the solid, $\bar{h} Z$

$z$ Thermal impedance of the solid in the time domain

\section{Subscripts}

0 Outer (away from the fluid) wall position, $y=0$

$\infty$ At fluid free-stream conditions, $y \rightarrow \infty$

A Isofux outer wall boundary condition

a Inner (in contact with the fluid) wall position, $y=a$ and $\widetilde{y}=0$

b Bulk fluid parameter 
f In the fluid domain

I Isothermal outer wall boundary condition

in Inlet condition, $x=0$

$n n^{\text {th }}$ component in the frequency domain

s In the solid domain

w On the (inner) solid wall, $y=a$ and $\widetilde{y}=0$

\section{Operators}

$\overline{(\cdot)}$ Mean component of $(\cdot)$

$(\cdot)^{\prime}$ Fluctuating component of $(\cdot)$

$(\cdot)^{\prime \prime}$ Normalised root mean square (rms) fluctuation intensity of $(\cdot), \frac{\sigma_{(\cdot)}}{(\cdot)}$

$\widehat{(\cdot)}$ Amplitude of fluctuation of $(\cdot)$, when $(\cdot)$ is sinusoidal

$(\cdot)^{*}$ [Unless otherwise defined, c.f.: $a^{*}, C^{*}, d^{*}, U^{*}$ and $Z^{*}$ Normalised amplitude of fluctuation of sinusoidal $(\cdot), \frac{\widehat{(\cdot)}}{\overline{(\cdot)}}=\sqrt{2}(\cdot)^{\prime \prime}=\sqrt{2} \frac{\sigma_{(\cdot)}}{\overline{(\cdot)}}$

$\Re\{(\cdot)\}$ Real part of $(\cdot)$

$\frac{\mathrm{D}}{\mathrm{D} t}(\cdot)$ Convective derivative

$(\cdot) \star(\cdot)$ Circular convolution 


\section{Introduction}

With the exception of a small number of purely laminar steady flows, the vast majority of engineering flows are unsteady, either: (i) periodic, which can be 'pulsating' (non-zero mean flow) or 'oscillating' (zero mean flow); (ii) quasiperiodic; or (iii) aperiodic (broadband, stochastic, turbulent). The present paper, continuing from Ref. [1], examines unsteady, forced convective heat transfer in the absence of compressibility. Although earlier studies of convective heat transfer in various arrangements involving unsteady flows have provided information on their heat exchange behaviour over a range of conditions, they have not yet led to a consensus as to the most useful way to describe their heat exchange (or insulation) characteristics, nor have they provided a clear insight into the crucial effects of unsteadiness and conjugation on these characteristics. It is important to address these aspects of convective heat transfer that arise in unsteady problems. Furthermore, on a practical level, there is a lack of reference literature and data to which design engineers can turn to in order to predict the temperature or heat flux in standard geometries, as they would for steady heat transfer [2]. In the first instance one would be interested in time-averaged quantities.

Much of our current understanding of heat transfer in these flows has been motivated by the specific desire to optimise the performance of energy-conversion machines that involve an interchange between heat and work, resulting in simultaneous pressure, flow and heat transfer fluctuations. For instance, in reciprocating thermoacoustic machines (engines, coolers) and devices such as expanders, compressors and combustion engines, or even gas turbine blades, the goal is to improve the heat and work transfer characteristics, while avoiding thermal failure. A typical example of this is encountered in Stirling engine regenerators (stacks, foams, porous media), where the aim is to maximise the heat transfer between the gas and the solid regenerator material while minimising the pressure drop in the flow through the regenerator. Nevertheless, energy-conversion devices involve for the most part unsteady heat transfer in the presence of compressible gaseous flows. In the treatment of most gaseous flows the assumption of a constant wall temperature is adequate [3] and the heat transfer process is not conjugate. These problems are simplified to obtaining information on the heat transfer coefficient (HTC) and using this to quantify the convective heat transfer in the fluid. The effect of conduction in the solid and hence of any solid parameters (i.e. thermophysical properties and geometric variables) drop out of this problem. On the other hand compressibility introduces an additional complication into the problem arising from the contribution of work towards the total energy budget; in fact, a consensus as to the effect of unsteadiness (i.e. pulsation/oscillation frequency) on the HTC continues to escape the community due to contradicting findings [4].

Even in simple incompressible flows where pressure work is absent, the question of whether the heat exchange in an unsteady flow is higher or lower than that of the equivalent (time-averaged) steady flow with otherwise similar conditions has not been answered definitively, despite numerous targeted and carefully performed experimental investigations, analytical studies and numerical simulations. Sorin et al. [5] measured a 
HTC in the intermittent flow in an exhaust pipe that was consistently higher than the steady flow equivalent, increasing with increasing frequency, reaching a maximum and decreasing as the frequency was increased further. Conversely, the experimental and numerical study performed in an incompressible oscillatory pipe flow with uniform heat flux by Zhao and Cheng [4] demonstrated a monotonic increase in the HTC with increasing dimensionless frequency, in agreement with the experiments of Leong and Jin [6] performed in a foam-filled channel. Yu et al. [7] solved analytically the heat transfer problem in an incompressible pulsating pipe flow with uniform heat flux. Interestingly, the authors reported no effect on the time-averaged HTC due to pulsation. By contrast, Habib et al. [8] reported a complex non-monotonic relationship between the HTC and the frequency in pulsating pipe flow experiments with a constant heat flux boundary condition (B/C). Generally, previous studies can be classified into four groups, according to which flow pulsations can either: (i) enhance [9, 10]; (ii) deteriorate [11]; (iii) have no effect on [12, 13]; or (iv) have a complex effect (i.e. enhance or deteriorate) on heat transfer depending on the character of the flow $[14,15]$.

A host of processes are known to exhibit unsteady periodic or turbulent fluid flow. An exchange of heat between the fluid and any containing solid walls in these problems can result in some form of unsteady and conjugate heat transfer, that in turn can give rise to the issues described in Ref. [1] (Section 1). It would be useful to develop a simple and efficient modelling tool for the engineering prediction of these processes and the design of related systems and devices, so that the effects of material properties and geometry can be easily understood and studied. In our related paper (Ref. [1]) a framework involving the use of a time-varying HTC was presented for analysing and modelling unsteady and conjugate (fluid-solid) heat transfer processes. An algorithm was developed for solving the temperatures and heat fluxes to satisfy both Newton's law of cooling in the fluid domain and the one-dimensional (1-D) unsteady heat diffusion (conduction) equation in the adjacent solid domain; the latter via the derivation of a thermal transfer response. With this approach an arbitrary HTC can be prescribed and the temperature and heat flux responses of the fluid can be found for various convecting flows by integrating the 1-D energy equation in the streamwise direction.

In the present paper the analysis presented in Ref. [1] is applied to two fundamental non-isothermal/diabatic flow problems and compared to experimental results: (i) heated falling film flows, where a thin layer of liquid flows due to gravity over an electrically heated, vertically inclined flat plate, in which interfacial waves are induced on the free surface of the film either naturally or forced through pulsation, which impart an unsteady heat transfer on the plate; and (ii) a separated flow downstream of a backwards-facing step, in which the resulting turbulent recirculating region behind the step and oscillations of the flow reattachment position result in unsteady heat transfer in that region (with data provided by Nakamura [16-18]). The characteristics of the HTC in the two cases are fundamentally different. In the film flow the low-dimensional waves are regular and exhibit periodicity, which results in a periodic fluctuation in the HTC. On the other hand, in the backwards-facing step the HTC is broadband in nature due to the flow separation and turbulence. 


\section{Case Study I: Heated Falling Films}

Film flows with periodic (externally excited) or stochastic interfacial wave structures have been studied extensively. Lel et al. [19] performed point measurements with high temporal resolution and accuracy of film thickness and interfacial wave velocity using a confocal lens technique and compared it to a fluorescence intensity method, and reported satisfactory agreement. Further, Moran et al. [20] made local measurements of the velocity profile within the liquid film as hydrodynamic waves travel down the film surface, and found that for large waves the velocity profiles were significantly different to Nusselt theory [21]. These flows are of interest to the present work, since: (i) the interfacial wave dynamics arising from hydrodynamic instabilities, and the associated varying liquid film thickness, will give rise naturally to spatiotemporally varying HTCs; and further (ii) temperature gradients induced within the film, as this undergoes heating or cooling, can result in significant surface tension gradients and give rise to thermocapillary 'Marangoni' instabilities [2224], which will also affect the interfacial, flow and heat transfer processes. Generally thinner films exhibit the largest heat and mass transfer rates, however in very thin films these instabilities can lead to dewetting or dry spot formation, which will degrade the overall heat and mass transfer characteristics.

The temperature of a fluid film can be measured with laser fluorescence techniques, which have allowed non-intrusive temperature measurements with accuracies better than $0.5 \mathrm{~K}$ to be achieved. Such a method is employed herein, by using the temperature-sensitive dye Rhodamine B. Fluorescence methods have proven popular in evaluating the heat exchange of microchannel devices [26, 29], in plate heat exchangers with oscillating flow $[27,28]$ and has also been used to measure fluid film temperatures elsewhere [30, 31]. In particular, Schagen et al. [30] and, Schagen and Modigell [31] employed a combined ultraviolet (UV) fluorescence-phosphorescence technique with a Diacetyl marker at a single point in the flow. The authors used these measurements to recover simultaneous information on the liquid film thickness and temperature profile within the film, and hence the HTC, at the position of the point measurement. However, these measurements were recovered by assuming a Nusselt velocity profile in the film, which may not account for deviations from this theoretical flow (e.g. as reported by Moran et al. [20]), due to two or three-dimensional flow effects or unsteadiness (including turbulence) in the film.

The free-surface temperature of liquid films has been measured directly using infrared (IR) thermography, sometimes with an IR filter placed in front of the camera lens to block the wavelengths of radiation to which the working fluid is transparent. The key aspect of this measurement is the absorptivity of the fluid, which is required to be high at the wavelengths used for the thermography. This ensures the observation of a thin layer of fluid near the interface (i.e. of the free surface), without contamination from the bulk of the fluid deeper within the film, or from the solid substrate. For example, Lel et al. [19, 32] used an IR camera to measure the interfacial temperature of silicone oil films. Silicon fluids were selected as they allowed a wide range of viscosities to be spanned, with a corresponding range of Reynolds and Kapitza numbers. In 
this case the absorption coefficient allowed $99 \%$ of the radiation to be absorbed within a thin $(10-15 \mu \mathrm{m})$ layer at the surface of the films [32]. IR thermography has also been used extensively in water film flows (e.g. [33, 34], and many others), and is also employed in the present measurements.

\subsection{Experimental Method}

An experiment was conducted to measure the instantaneous temperature and heat flux of a liquid (water) film as it flows down a flat, inclined heated foil. The foil was made from $99.6+\%$ pure titanium and had a thickness $a$ of $50 \mu \mathrm{m}$. Heating was achieved by holding the foil tight between two $10 \mathrm{~mm}$ thick copper electrodes, and applying a current from a DC power supply. The apparatus was capable of a maximum heat flux of $4.2 \mathrm{~W} / \mathrm{cm}^{2}$ over a $1200 \mathrm{~cm}^{2}$ area. At a distance of $300 \mathrm{~mm}$ upstream of the imaged area the water was distributed over the foil through a $w=300 \mathrm{~mm}$ wide knife-edge slot to form a thin fluid film with a uniform thickness across the heated area. The water flow that fed the distributor was a combination of two separate supplies. One was a steady supply whose flow-rate was measured by rotameters. The second, through which a prescribed proportion of total flow-rate was by-passed, was a pulsating supply, modulated by a rotating throttle valve to excite coherent large-scale waves that flowed down the foil at a controlled frequency. A simplified schematic of the flow and measurement arrangement is shown in Fig. 1.

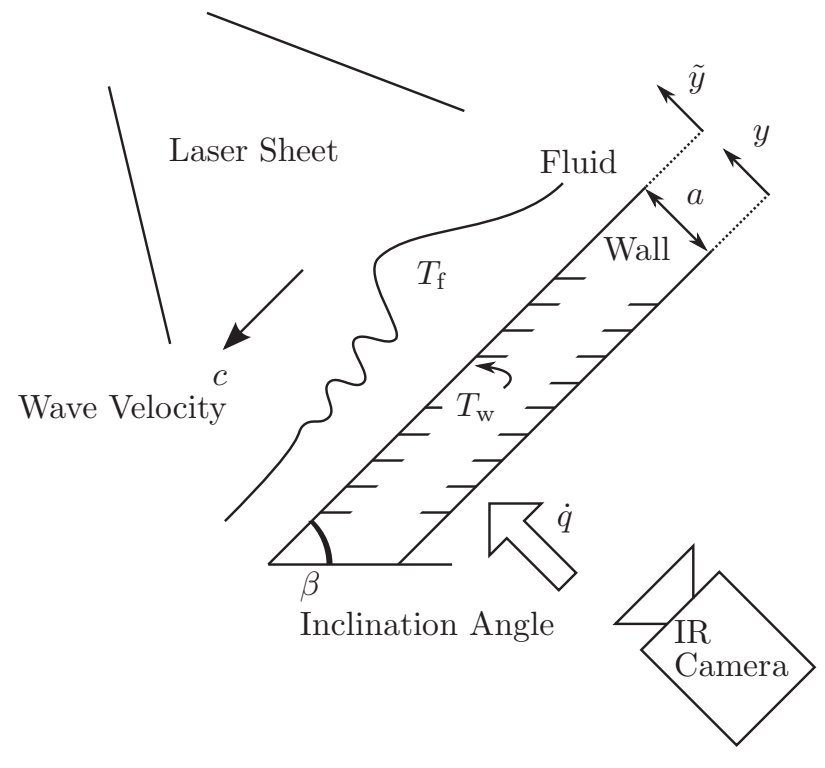

Figure 1: Diagram of the film flow experiment.

Having established a flow condition, a laser-induced fluorescence (LIF) technique was then used to measure the film thickness and free-surface temperature simultaneously. The water phase was marked by predissolving a temperature-dependent fluorescent dye, Rhodamine B, to form a perfectly mixed aqueous-dye 


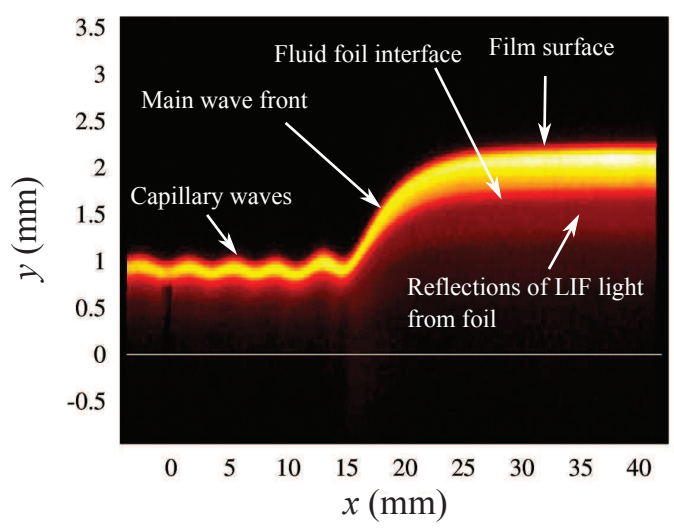

Figure 2: Detailed view of a wave in a LIF image.

solution of low concentration $(\sim 1 \mathrm{~g} / \mathrm{L})$. The aqueous liquid was excited by a light sheet of thickness $100 \mu \mathrm{m}$ that was generated by a dedicated sheet optics lens combination. The sheet optics combination was connected, via a guiding arm, to a $532 \mathrm{~nm}$ Nd:YAG laser that produced pulses of light at $100 \mathrm{~Hz}$. The resulting fluorescent light was captured by a pair of CMOS cameras which were synchronised to capture images upon each laser pulse. A pair of cameras were used to increase the field of view of the LIF image and in addition the second camera was utilised to monitor the laser energy of each laser pulse. The cameras were arranged to be perpendicular to the laser sheet, and any perspective distortion was corrected for by using a calibration graticule to calculate the inverse transform of the image [25].

The captured images were then stored as an image sequence for later processing. An optical long pass dichroic filter was positioned in front of the camera lenses to block the incident laser light but pass the fluorescent light from the Rhodamine B dye. Based on the temperature dependence of the quantum efficiency of Rhodamine B fluorescence [26, 29], imaging of the emitted light allowed not only the identification of the interface height of the waves travelling down the film (i.e. film depth), but also the direct measurement of the temperature of the free-surface of the film. An example of a captured image of the wave is shown in Fig 2.

Since the intensity of the florescence emission from the dye is temperature dependant the film interface temperature was inferred from the intensity of the imaged film at the known interfacial location. Simultaneous to the capture of LIF images, temperature measurements were made on the underside of the foil using an IR camera (see Fig. 1) and in the fluid inlet with thermocouples. In addition, in a separate set of experiments, both the IR camera and a series of fine $(75 \mu \mathrm{m})$ thermocouples were used to make independent measurements of the film and foil temperatures for the purposes of calibration and verification.

The use of electrodes that were much thicker, and therefore with a considerably lower resistance than the foil, ensured that the power supplied was distributed evenly over the foil, so that the heating effect was 
uniform. This was checked by measuring the voltage distribution over the foil using point measurements with a multimeter. No noticeable voltage distortion was observed, with a worst case deviation in power of $0.1 \%$ over the $300 \times 400 \mathrm{~mm}$ foil area. Moreover, the uniform heating was confirmed by the observation of a uniform temperature distribution across the foil in dry test cases, wherein the foil is heated in the absence of any fluid flow and the foil temperature was measured with the IR camera. In these tests it was also found that convection of heat to the air was minimal, being less than $1 \%$ of the heat flux into the liquid that was experienced in the falling film flows, in worst case conditions ${ }^{1}$. Hence, it is important to note that, since the heat flux into the film at the inner wall (foil surface in contact with the fluid) is much much larger than the losses to the surroundings from the outer wall (un-wetted surface on the opposite, underside of the foil), the outer side of the foil can be considered as an adiabatic B/C. The liquid temperature was kept low, below $40^{\circ} \mathrm{C}$, to minimise evaporation and to focus the investigation on the forced convective heat transfer processes in liquid films.

Table 1: Thermal properties of materials and fluid in the film flow experiments.

\begin{tabular}{lccc}
\hline & $\begin{array}{c}\text { Density, } \rho \\
\left(\mathrm{kg} \mathrm{m}^{-3}\right)\end{array}$ & $\begin{array}{c}\text { Heat capacity, } c \\
\left(\mathrm{~J} \mathrm{~kg}^{-1} \mathrm{~K}^{-1}\right)\end{array}$ & $\begin{array}{c}\text { Thermal conductivity, } k \\
\left(\mathrm{~W} \mathrm{~m}^{-1} \mathrm{~K}^{-1}\right)\end{array}$ \\
\hline Water & 1000 & 4200 & 0.6 \\
Titanium & 4510 & 540 & 21.9 \\
\hline
\end{tabular}

The foil is thin, that is, the foil is over an order of magnitude thinner than any thermal feature observed on its surface with the IR camera. Consequently it can be assumed that heat transfer in the foil is dominated by 1-D thermal conduction through its thickness (refer to Ref. [18] for a full explanation of the spatial resolution of IR thermography measurements). With this assumption the model for 1-D thermal conduction in the solid (Ref. [1]) can be used to describe the foil's thermal response to temperature fluctuations. This response will depend upon the imposed outer wall boundary condition and, as it was stated previously, the thermal boundary condition on the back/underside surface of the foil (or, outer wall ' 0 ') can effectively be assumed to be adiabatic. Note that as, in the current problem, the solid diffusivity is constant and so the thermal conduction in the solid is linear: the problem of finite 1-D thermal conduction with an adiabatic boundary condition imposed at one surface and constant heat generation in the solid (through resistive heating) is equivalent to the system considered in Ref. [1] where an isoflux wall boundary condition is imposed at one end of the domain and with no heat generation in the solid. The resulting thermal response that relates the common fluid-solid interface temperature (inner wall ' $w$ ') to the measured temperature fluctuations on the

\footnotetext{
${ }^{1}$ Specifically it was found that, in the absence of a liquid film flow, $50 \mathrm{~W}$ of heat were required to maintain a steady temperature of $60{ }^{\circ} \mathrm{C}$ over the surface of the foil. However after establishing a typical water film flow over the foil, up to $5 \mathrm{~kW}$ were required to obtain the same foil temperatures.
} 
outer wall for a given frequency is (see Ref. [1] and Appendix Appendix A for a summary of the proof),

$$
\frac{T_{\mathrm{w}, n}}{T_{0, n}}=\cosh \left\{(1+\mathrm{i}) a_{n}^{*}\right\}
$$

while the corresponding heat flux response is given by,

$$
\frac{\dot{q}_{\mathrm{w}, n}}{T_{0, n}}=\frac{(1+\mathrm{i})}{\sqrt{2}} \epsilon_{\mathrm{s}} \sqrt{\omega_{n}} \sinh \left\{(1+\mathrm{i}) a_{n}^{*}\right\},
$$

where $a_{n}^{*}=a / \delta_{\mathrm{s}, n}$ is a normalised solid thickness with $a$ the foil thickness and $\delta_{\mathrm{s}, n}=\sqrt{2 k_{\mathrm{s}} / \rho_{\mathrm{s}} c_{\mathrm{s}} \omega_{n}}=$ $\sqrt{2 \alpha_{\mathrm{s}} / \omega_{n}}$ the thermal diffusion length in the solid associated with the $n^{\text {th }}$ harmonic of the thermal fluctuations, $\alpha_{\mathrm{s}}$ is the thermal diffusivity and $\epsilon_{\mathrm{s}}=\sqrt{k_{\mathrm{s}} \rho_{\mathrm{s}} c_{\mathrm{s}}}$ the thermal effusivity of the solid material.

By combining Eq. (1) and Eq. (2) the thermal impedance of the solid as seen by the fluid is,

$$
\frac{\dot{q}_{\mathrm{w}, n}}{T_{\mathrm{w}, n}}=\frac{1}{Z_{\mathrm{A}}\left(\omega_{n}\right)}=\frac{(1+\mathrm{i})}{\sqrt{2}} \epsilon_{\mathrm{s}} \sqrt{\omega_{n}} \tanh \left\{(1+\mathrm{i}) a_{n}^{*}\right\} .
$$

Thus, the temperature at the inner wall $T_{\mathrm{w}}$ can be found by implementing an inverse filter with the response in Eq. (1) on the temperature of the foil measured on the outer wall by the IR camera, $T_{0}$. Similarly the instantaneous heat flux at the inner wall $\dot{q}_{\mathrm{w}}$ can be evaluated from the IR temperature measurement, the response in Eq. (2) and the electrical power supplied to the foil. Eq. (1) indicates (after using the material properties listed in Table 1) that the attenuation in the amplitude of the temperature fluctuations through the foil thickness at $50 \mathrm{~Hz}$, which is the Nyquist frequency for the image capture rate, is $0.1 \%$.

\subsection{Experimental Results}

Typical results can be seen in Fig. 3. The film interface height $d$, temperature $T_{\mathrm{f}}$, and heat flux at the common fluid-solid surface (inner wall) $\dot{q}_{\mathrm{w}}$, all vary with time and spatial position within the imaged area. Note that the local, time-varying heat flux $\dot{q}_{\mathrm{w}}$ is defined herein as +ve from the fluid into the solid wall. However in Fig. 3 the magnitude of the heat flux is displayed as heat is being applied from the foil (solid wall) to the fluid. The instantaneous HTC $h$, is also evaluated from these data and shown. In Fig. 3(a) several spatial snapshots at different time instances are shown as a wave progresses through the $40 \mathrm{~mm}$ long interrogation (viewing) area. The main wave front can be clearly seen, and is proceeded by several, smaller capillary waves. Where the film is thickest, the temperature difference between the water and the foil is largest, and where the film is thin the temperature difference is small. Conversely the HTC is lowest where and when the film is thick and much higher when it is thin, as we would expect (see Eq. (10)). Figure 3(b) shows how the heat flux $\dot{q}_{\mathrm{w}}$ rises and falls locally with each passing wave. In this case the fluctuation in $\dot{q}_{\mathrm{w}}$ has an amplitude of $0.6 \%$ of the mean heat flux. In correspondence to the wall heat flux, the wall temperature $T_{\mathrm{w}}$ is also approximately constant. The majority of the fluctuations in the bulk fluid temperature difference $\Delta T_{\mathrm{f}}=T_{\mathrm{f}}-T_{\mathrm{w}}$ in this case emerges from fluctuations in the bulk fluid temperature $T_{\mathrm{f}}$. This result arises in 
measurements taken far downstream of the inlet; closer to the fluid inlet considerably larger fluctuations in $T_{\mathrm{w}}$ and $\dot{q}_{\mathrm{w}}$ were found.
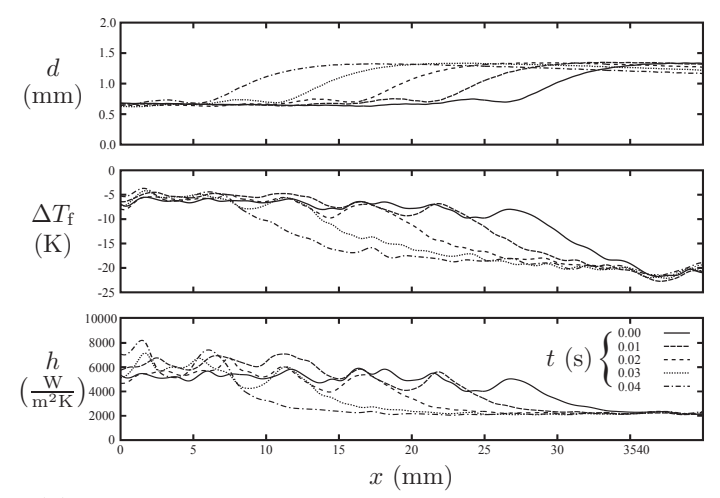

(a) Snapshots of the profile at different times instances

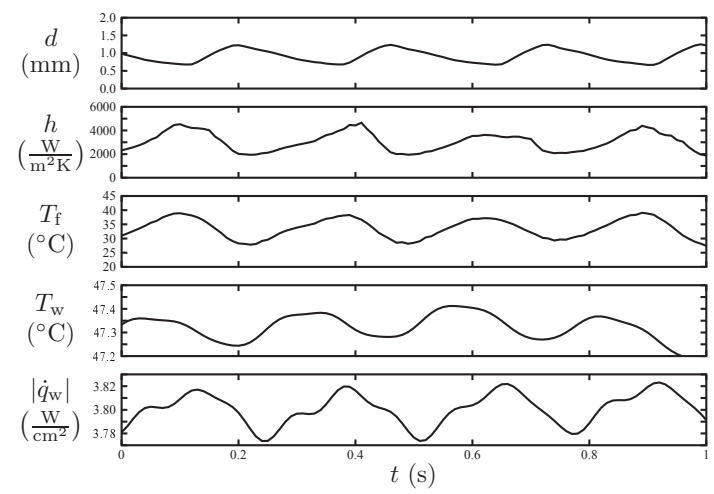

(b) Time evolution.

Figure 3: Profile of a wave and the resulting heat transfer as the wave flows down the heated plate, for the flow conditions of $\operatorname{Re}=300, \operatorname{Pr}=5.0$ and $a_{1}^{*}=a / \delta_{\mathrm{s}, 1}=0.056$.

The flow of a steady, fully developed, laminar liquid film on an inclined plate is described by the Nusselt film flow solution [21]. Nusselt flow forms a natural basis for comparison of the heat transfer measured in the wavy film flows investigated in the present experiments, although differences should be expected since our flows are not steady, or laminar at higher Reynolds numbers, and also may not be fully developed by the measurement location. The HTC in a steady and fully developed Nusselt flow can be obtained by considering that the body forces acting on the fluid due to gravity must be in balance with the viscous forces due to shear across the film, such that the fluid velocity profile satisfies the analytical Nusselt solution [21],

$$
U_{x}=\frac{g \sin \beta}{\nu_{\mathrm{f}}} \widetilde{y}\left(d-\frac{\widetilde{y}}{2}\right)
$$

which can be re-written in terms of Reynolds number $R e$, or the bulk film velocity $U_{\mathrm{b}}$ as,

$$
U_{x}=\frac{3 \nu_{\mathrm{f}} R e}{d}\left(\frac{\widetilde{y}}{d}\right)\left[1-\frac{1}{2}\left(\frac{\widetilde{y}}{d}\right)\right] ; \quad \frac{U_{x}}{U_{\mathrm{b}}}=3\left(\frac{\widetilde{y}}{d}\right)\left[1-\frac{1}{2}\left(\frac{\widetilde{y}}{d}\right)\right],
$$

where $g$ is gravitational acceleration and $\nu_{\mathrm{f}}$ the kinematic viscosity of the fluid. Here, the coordinate $\widetilde{y}=y-a$ is perpendicular to and defined as zero at the inner wall. The Reynolds number has been obtained from $R e=\int_{0}^{d} U_{x} d \widetilde{y} / \nu_{\mathrm{f}}=U_{\mathrm{b}} d / \nu_{\mathrm{f}}=\Gamma / \nu_{\mathrm{f}}$ where $\Gamma$ is the fluid flow-rate per unit channel width $Q / w$, such that $R e=g \sin \beta d^{3} / 3 \nu_{\mathrm{f}}^{2}$, and the bulk flow velocity of the film is given $U_{\mathrm{b}}=g \sin \beta d^{2} / 3 \nu_{\mathrm{f}}$.

Now, writing down the energy equation in the fluid,

$$
\frac{\mathrm{D} T_{\mathrm{f}}}{\mathrm{D} t}=\alpha_{\mathrm{f}} \nabla^{2} T_{\mathrm{f}}
$$

and noting that the flow is thin, fully developed, steady-state and laminar allows some of the terms in Eq. (6) to be neglected. Furthermore, for a constant heat flux B/C the flow will be self-similar in the streamwise 
direction, which means that the streamwise temperature gradient $\partial T_{\mathrm{f}} / \partial x$ will be constant. On applying these simplifications, the governing equation of the temperature profile in the film becomes,

$$
U_{x} \frac{\partial T_{\mathrm{f}}}{\partial x}=\alpha_{\mathrm{f}} \frac{\partial^{2} T_{\mathrm{f}}}{\partial \widetilde{y}^{2}} .
$$

Substituting Eq. (5) into Eq. (7) and integrating subject to the B/Cs $\partial T_{\mathrm{f}} / \partial \widetilde{y}(\widetilde{y}=0)=-\dot{q}_{\mathrm{w}} / k_{\mathrm{f}}$ on the solid wall and $\partial T_{\mathrm{f}} / \partial \widetilde{y}(\widetilde{y}=d)=0$ at the free surface, while noting that $\dot{q}_{\mathrm{w}}=-U_{\mathrm{b}} \rho_{\mathrm{f}} c_{\mathrm{f}} d\left(\partial T_{\mathrm{f}} / \partial x\right)$ in the fluid with $\dot{q}_{\mathrm{w}}$ defined as + ve from the fluid to the wall, we obtain the following temperature profile in the film,

$$
T_{\mathrm{f}}=T_{\mathrm{w}}+\frac{\dot{q}_{\mathrm{w}}}{k_{\mathrm{f}}} d\left[-\frac{1}{8}\left(\frac{\widetilde{y}}{d}\right)^{4}+\frac{1}{2}\left(\frac{\widetilde{y}}{d}\right)^{3}-\left(\frac{\widetilde{y}}{d}\right)\right] .
$$

from which the bulk temperature is thus,

$$
T_{\mathrm{b}}=\frac{1}{d} \int_{0}^{d} T_{\mathrm{f}} d \widetilde{y}=T_{\mathrm{w}}+\frac{2}{5} \frac{\dot{q}_{\mathrm{w}}}{k_{\mathrm{f}}} d
$$

which gives a film Nusselt number $N u$ of,

$$
N u=\frac{h d}{k_{\mathrm{f}}}=2.5
$$

By comparing this value of Nusselt number $N u$ to that corresponding to the measured HTCs from our film flows, it is possible to investigate whether the waves in the film flows result in enhanced or reduced heat transfer compared to Nusselt flow. It is expected that the waves will increase mixing, by inducing unsteadiness in the flow and turbulence at higher $R e$, enhancing heat transport and resulting in a higher $N u$ than that in Eq. (10). Other effects may also occur, for example the unsteadiness of the waves may result in a velocity profiles different from those described by Eq. (5), which in turn would result in a different temperature profile in the film. In any case, this comparison can give an indication of how well the investigated film flows can be described by the idealised steady-state Nusselt flow.

Fig. 4 shows joint probability density function (PDF) contours of the instantaneous, local HTC $h$ and corresponding instantaneous, local film thickness $d$ at the measurement location for a particular flow condition. Contours of higher probability indicate that the HTC and film thickness were more likely to be in the region enclosed by that curve. A definite inverse relationship between the instantaneous HTC and the film thickness emerges. Further, the curves from each different foil heat input setting lie on top of each other, meaning that the measured HTC is independent of the heat flux applied ${ }^{2}$. The independence of the HTC from the applied heat flux for a given flow condition is important as it validates the usefulness of the definition of a local and instantaneous HTC, and enables its meaningful application to the description (and the subsequent comparison) of heat transfer in different flow conditions ${ }^{3}$.

\footnotetext{
${ }^{2}$ However, even though $h$ has been shown to be independent of $\dot{q}_{\mathrm{w}}$, the converse (not shown here) is not true; at a given flow
} 

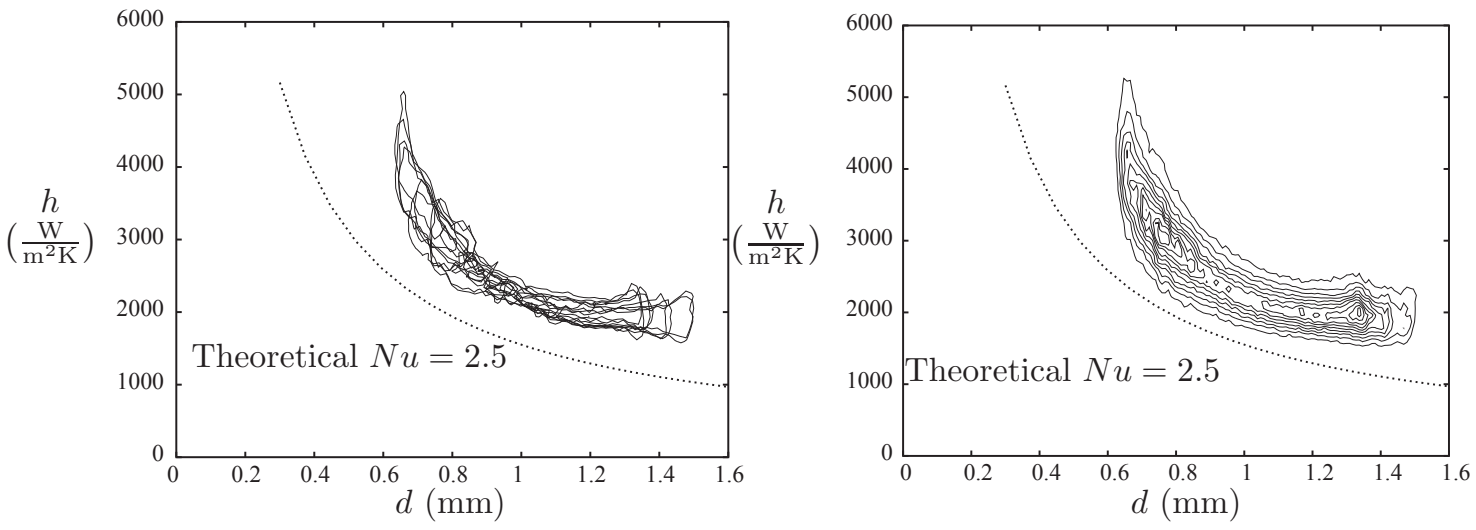

(a) Overlay of $50 \%$ probability joint-PDF contours of HTC $h$ and film thickness $d$, one contour extracted per run of $\dot{q}_{\mathrm{w}}$ setting, with each contour signifying the region within which $h$ and $d$ lay for $\geq 50 \%$ of the time in that particular run.

(b) Joint-PDF contours of $h$ and $d$ compiled over the whole set of 12 runs with different (uniform, steady) heat inputs to the foil substrate $\bar{q}_{\mathrm{w}}$ from $200 \mathrm{~W}$ to $5 \mathrm{~kW}$, but the same flow conditions. The data from all runs are combined.

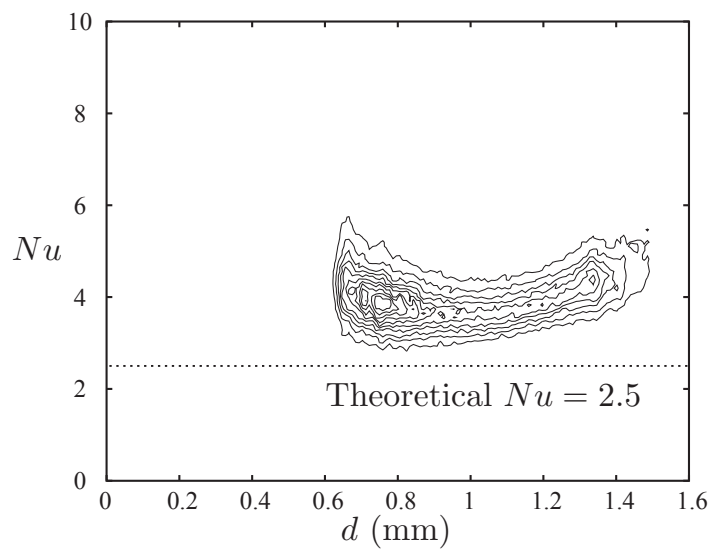

(c) Joint-PDF of $N u$ and $d$ corresponding to (b).

Figure 4: Instantaneous HTC characteristics for a set of 12 runs with the same flow condition $R e=300$, $\operatorname{Pr}=5.0$ and $a_{1}^{*}=0.056$, but with different (uniform, steady) heat inputs $\overline{\dot{q}}_{\mathrm{w}}$ to the foil substrate over the range $200 \mathrm{~W}$ to $5 \mathrm{~kW}$. Showing joint probability density functions (PDFs) of the dependence of instantaneous local $h$ on the instantaneous local film thickness $d$. 
Also shown in Fig. 4(a) and in the combined results in Fig. 4(b) is the theoretical HTC obtained for Nusselt film flow, corresponding to $N u=2.5$ from Eq. (10). The experimental data and the theoretical Nusselt result are in good agreement. The measured HTC follows closely an inversely proportional relationship with the film thickness, showing slight deviations when the films are at their smallest and largest thickness. Generally, the magnitude of the measured HTC is only slightly larger than that predicted by Nusselt theory, though the experiments do show consistently elevated values compared to the theoretical result. The enhanced heat transfer relative to a purely steady laminar Nusselt flow is probably a direct consequence of increased mixing and transport within the films from the passing of the wave fronts and the unsteadiness of the velocity profile in the fluid. A higher HTC would also be expected if the flow is still developing. However the measurements were made a distance $\sim 300 d_{0}$ initial film thicknesses downstream of the inlet, whereas a standard relation for the thermal development length in the idealised Nusselt flow, $L_{\mathrm{th}} / d=0.03 R e \operatorname{Pr}$, gives an estimate of the development length of approximately $60 \mathrm{~d}$ fluid thicknesses.

Fig. 4(c) shows the same data as Fig. 4(b), but this time in relation to the Nusselt number $N u$ instead of the HTC $h$ plotted against $d$. Generally $N u$ is slightly greater than the theoretical Nusselt value of 2.5, with the difference between the two increasing when the film height is at its thickest, and at its thinnest. A possible explanation for the increased $N u$ when the film is thick may concern the increased flow (velocity component) in the normal direction to the wall and the increased space for mixing under the waves, and perhaps, an increase in the turbulence behind the wave front leading to a heat transfer enhancement. Conversely, the enhancement when the film is thin may be due to the modification of the temperature and velocity profiles in the film due to the unsteadiness in the flow [20], resulting in increased thermal gradients at the wall.

\subsection{Analysis and Modelling}

In our previous paper a framework was developed for the modelling of unsteady and conjugate heat transfer problems [1]. Firstly, the analytical solution to 1-D conductive heat transfer in a finite solid domain is used to find a thermal impedance of the solid. The heat transfer in various 1-D convecting flows is then modelled by using this transfer function as a boundary condition to the fluid domain. Once a time-varying periodic HTC is imposed, the wall heat flux and temperatures are found numerically by simultaneously solving Newton's law of cooling, that describes the forced convective heat transfer in the fluid, and the aforementioned transfer function governing the heat conduction in the solid. Energy conservation in the fluid is used to calculate the evolving temperature of the fluid within the domain from an initial condition, and thus the transfer function relating the temperature of the fluid and the heat flux at the wall. This model is then used to examine the

\footnotetext{
condition $\Delta T_{\mathrm{f}}$ and $\dot{q}_{\mathrm{w}}$ are both dependent on $h$.

${ }^{3}$ There are instances when this independence can be lost, and the heat flux can affect the HTC. For example, this can occur if the heat flux is high enough to instigate boiling, or to give rise to significant gradients in the fluid properties (e.g. viscosity, or density such as in naturally convecting flows).
} 
effects of the dimensionless wall thickness $a^{*}=a / \delta_{\mathrm{s}}$, the Biot number $B i=\bar{h} a / k_{\mathrm{s}}$, the HTC fluctuation intensity $h^{\prime \prime}=\sigma_{h} / \bar{h}$, and the heat capacity ratio $C_{\mathrm{R}}=\left(\rho_{\mathrm{f}} c_{\mathrm{f}} / \rho_{\mathrm{s}} c_{\mathrm{s}}\right)(d / a)$ on the heat transfer augmentation ratio,

$$
A=\frac{\overline{\dot{q}}_{\mathrm{w}}}{\bar{h} \overline{\Delta T_{\mathrm{f}}}}=1+\overline{\overline{h^{\prime}}} \frac{\Delta T_{\mathrm{f}}^{\prime}}{\overline{\overline{\Delta T_{\mathrm{f}}}}} .
$$

In the present paper we investigate the predictive ability of the modelling framework developed in Ref. [1] with respect to the direct measurements of augmentation in the heated falling film flow experiments that were described above. To this end, the measured HTC in each experimental run, defined by a prescribed set of flow and heating conditions, is approximated by a pure sinusoid, $h=\bar{h}+\widehat{h} \sin (\omega t)$, such that $\widehat{h}=\sqrt{2} \sigma_{h}$, and the known parameters $a^{*}, B i, h^{\prime \prime}=h^{*} / \sqrt{2}$ and $C_{\mathrm{R}}$ from each experimental run are supplied to the above model and the predicted and measured augmentation ratios $A$ are compared.

In the film flow experiments it is expected that on average the temperature of the fluid will increase linearly in the downstream direction as it is heated by the foil. Also perturbations in the fluid temperature, due to fluctuations in the film HTC or waves, will convect downstream while growing or decaying as seen in Case V in Ref. [1]. However, unlike Case V where the HTC was modelled as changing instantaneously but uniformly over the entire domain, here the HTC will convect, in phase, with the fluid waves as these travel down the foil. Due to this convection of the fluctuations in the HTC with the fluid, the coupling between the fluid temperature and the HTC will become stronger with increasing distance from the initial condition, i.e. the fluid inlet.

By taking the energy equation in the fluid (Eq. (6)) and integrating in the direction normal to the heated surface, the response of the bulk film temperature $T_{\mathrm{f}, \mathrm{b}}$ to a wall heat flux $\dot{q}_{\mathrm{w}}$ can be approximated by,

$$
\rho_{\mathrm{f}} c_{\mathrm{f}} d \frac{\mathrm{D} T_{\mathrm{f}, \mathrm{b}}}{\mathrm{D} t}=-\dot{q}_{\mathrm{w}}
$$

If a constant flow velocity is assumed Eq. (6) can be solved by the method of characteristics. The governing equation of the fluid film temperature is then found to be,

$$
\frac{\partial T_{\mathrm{f}, \mathrm{b}}}{\partial c}=-\frac{h\left(t_{0}\right)}{\rho_{\mathrm{f}} c_{\mathrm{f}} d}\left(T_{\mathrm{f}, \mathrm{b}}-\rho_{\mathrm{f}} c_{\mathrm{f}} d\left[z\left(t_{0}\right) * \frac{\partial T_{\mathrm{f}, \mathrm{b}}}{\partial c}\right]\right),
$$

where $c$ is a characteristic line of a fluid element and $t_{0}$ is the initial time when the fluid element was at the inlet. Since the HTC is modelled as convecting with the fluid element it is only a function of $t_{0}$.

Eq. (13) is nonlinear due to the convolution with the solid response $z\left(t_{0}\right)$, which is the time-domain representation of the thermal impedance of the solid $Z\left(\omega_{n}\right)$. The equation can be solved numerically, though this is increasingly time consuming for regions a long way from the fluid inlet. Still, numerical experimentation reveals that the thermal solution of the fluid will start as being a response representative of an isothermal condition on the inner wall, in which $T_{\mathrm{w}}^{\prime}=0$ at the inlet (a stiff response), and tend to a response representative of an isoflux (but not adiabatic, $\overline{\dot{q}}_{\mathrm{w}} \neq 0$ ) condition on the inner wall, in which $\dot{q}_{\mathrm{w}}^{\prime}=0$ 
when the flow is fully thermally developed (a soft response). It is also found that a reasonable approximation can be made for the transfer function of the fluid by taking the transfer function from Case V [1] and adding a scale factor to the $C_{\mathrm{R}}$ to match this change in response. The modified fluid temperature response becomes,

$$
T_{\mathrm{f}, \mathrm{b}}=\bar{T}_{\mathrm{f}, \mathrm{b}}+\frac{B i a_{1}^{*}}{2 C_{\mathrm{R}} U^{*}} \frac{x}{a} \Re\left\{\frac{1}{\mathrm{i}} \sum_{n} \frac{1}{Z_{n}^{*} a_{n}^{*}} T_{\mathrm{w}, n} e^{\mathrm{i} \omega_{n} t}\right\},
$$

where the Biot number is based on the time-averaged HTC such that $B i=\bar{h} a / k_{\mathrm{s}}$, the dimensionless fluid velocity is $U^{*}=\left(\nu_{\mathrm{f}} / \omega d^{2}\right) R e$ and $R e=U_{\mathrm{b}} d / \nu_{\mathrm{f}}$, the dimensionless foil thickness for a given harmonic is $a_{n}^{*}=a / \delta_{\mathrm{s}, n}$, and the heat capacity ratio between the fluid film and the foil is $C_{\mathrm{R}}=\left(\rho_{\mathrm{f}} c_{\mathrm{f}} / \rho_{\mathrm{s}} c_{\mathrm{s}}\right)(d / a)$. Also note that the thermal impedance of the solid has been normalised by the mean HTC to give $Z_{n}^{*}=\bar{h} Z\left(\omega_{n}\right)$.

The heat transfer problem can now be solved to calculate the time-varying inner wall temperature $T_{\mathrm{w}}$ and heat flux $\dot{q}_{\mathrm{w}}$, given an prescribed fluctuation in the HTC. As the set of equations is nonlinear, a numerical method is employed to find the solution, in which the variables are discretised at regular steps in the time domain. First an arbitrary wall temperature, $T_{\mathrm{w}}$, is picked across the time domain. The fluid temperature $T_{\mathrm{f}}$ is then found from the fluid domain response in Eq. (14) using this wall temperature. Furthermore the wall heat flux, $\dot{q}_{\mathrm{w}}$, can be calculated from Newton's law of cooling, using the known imposed HTC and the temperature difference across the fluid film, $\Delta T_{\mathrm{f}}=T_{\mathrm{f}}-T_{\mathrm{w}}$. Similarly the wall heat flux, $\dot{q}_{\mathrm{w}}$, can also be calculated from the solid response in Eq. (3), using a fast Fourier transform to switch between the frequency and time domains. A nonlinear multidimensional equation solver can be used to solve for $T_{\mathrm{w}}$ so that the heat fluxes calculated by both methods are equivalent. Any nonlinear equation solver can be used and many computational libraries are capable of implementing fast algorithms for such solvers. An Euler stepping method can be slow and possibly unstable, so a Newton-Raphson like method was used, as implemented by Matlab's inbuilt fsolve function which chooses from a selection of algorithms (Fortran MINPACK library). Once a solution for $T_{\mathrm{w}}$ has been found $\dot{q}_{\mathrm{w}}$ and $T_{\mathrm{f}}$ can be calculated as before.

Figure 5 shows individual data points (one per experimental run) from two sets of experiments with different dimensionless foil thickness, or different fundamental (forcing) wave frequencies $a_{1}^{*}=a / \delta_{\mathrm{s}, 1}$. The measured nonlinear augmentation ratio $A$ is displayed against the HTC fluctuation intensity $h^{\prime \prime}=\sigma_{h} / \bar{h}$, and the Biot number $B i$ based on the mean HTC magnitude $\bar{h}$. A surface of the predicted augmentation from our theoretical model is also shown. The points generally follow the surface trend described by the model. We observe that, in the film flow experiments, an augmentation effect up to a $10 \%$ is observed when the Biot number $B i$ and $h^{\prime \prime}$ are at their largest respective values. The values of $h^{\prime \prime}$ and $B i$ were varied by the liquid film mean flow-rate and pulsation amplitude setting in each run. On comparing the two plots, one must consider that the amplitude of the waves was attenuated at higher frequencies (i.e. higher $a_{1}^{*}$ ), leading to depreciated fluctuation intensities at the higher wave frequencies.

A better picture of the augmentation behaviour predicted by the model, and the region of the parameter 


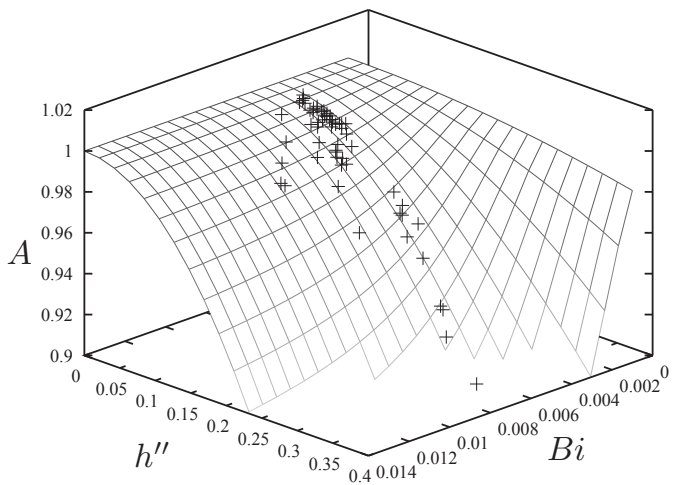

(a) $a_{1}^{*}=0.056$.

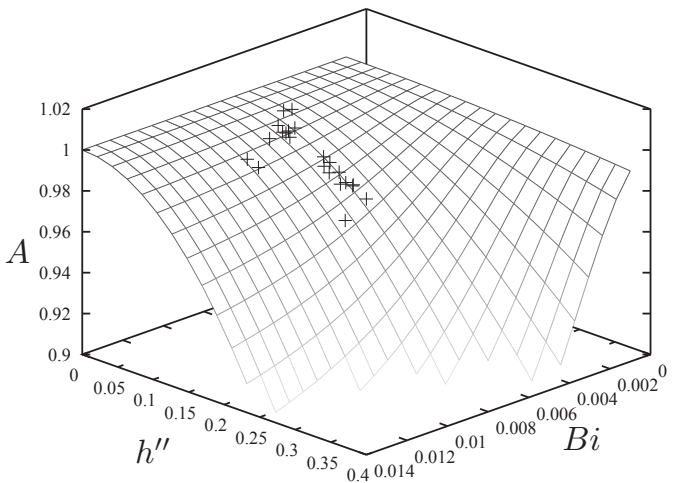

(b) $a_{1}^{*}=0.080$.

Figure 5: A view of the augmentation domain with experimental points.

space that has been spanned by the experimental data is shown in Fig. 6. Interestingly, the model predicts that at high $B i$ the degree of augmentation is dependent only on the HTC fluctuation intensity $h^{\prime \prime}$, and becomes independent of the mean $\mathrm{Bi}$. In this region of the map the wall heat flux and also the wall temperature are constant, such that the heat transfer problem is predominantly non-conjugate. The response of the augmentation ratio tends to that described by Eq. (17), which was derived for a constant inner wall heat flux (isoflux condition). This arises in these thermally developing flows due to the high values of the $B i$, which result in a strong coupling between the fluid temperature and the HTC at the measurement point, far downstream of the inlet, that act to constrain the fluctuations in the foil heat flux. At lower $B i$ the heat transfer problem becomes increasingly conjugate, with the wall temperature and heat flux responding increasingly to the fluctuations in the fluid. As the constraint on the foil heat flux is gradually removed, the increased conjugation gives rise to greater fluctuating foil temperatures and heat fluxes. This manifests in Fig. 6 as a rise in the contours of constant $A$, which implies that larger HTC fluctuation intensities $h^{\prime \prime}$ are required for the same level of $A$. The value of $B i$ at which this transition to conjugation occurs depends on the foil thickness and wave frequency. In the lower wave frequency data in Fig. 6(a) the transition occurs at lower $B i$ than in the higher wave frequency data in Fig. 6(b). In both cases the data from the experiments are straddling this transitional region, with $4 \times 10^{-3}<B i<9 \times 10^{-3}$.

The augmentation ratio $A$ is examined in terms of $B i$ in Fig. 7. Experimental data points (denoted by '+') are shown next to the modelled points (denoted by 'o'). The latter required the input of the values of $h^{\prime \prime}$ and $B i$, which were taken directly from the experiments. Generally a very good agreement is found between the experimental and predicted augmentation ratios. The value of $A$ decreases gradually with increasing $h^{\prime \prime}$, falling to about $90 \%$ in the experiments with the smaller dimensionless foil thickness $a_{1}^{*}=0.056$, and $97 \%$ in the experiments with the larger dimensionless foil thickness $a_{1}^{*}=0.080$. The theoretical prediction indicates that the deviation from unity accelerates at increasingly higher $h^{\prime \prime}$, suggesting that the accurate 


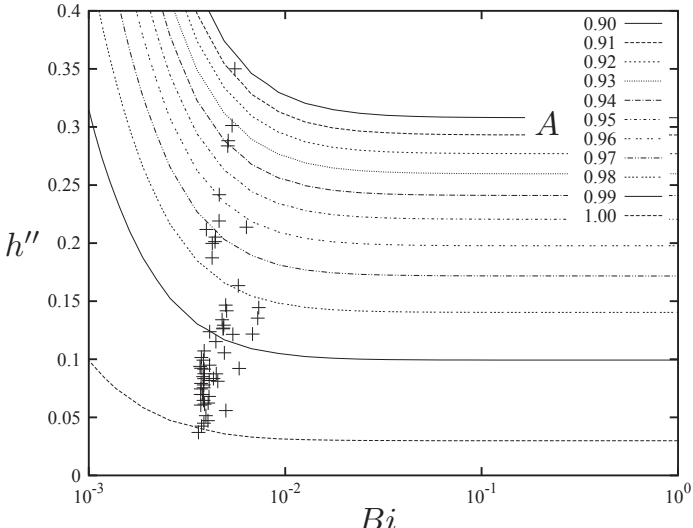

(a) $a_{1}^{*}=0.056$.

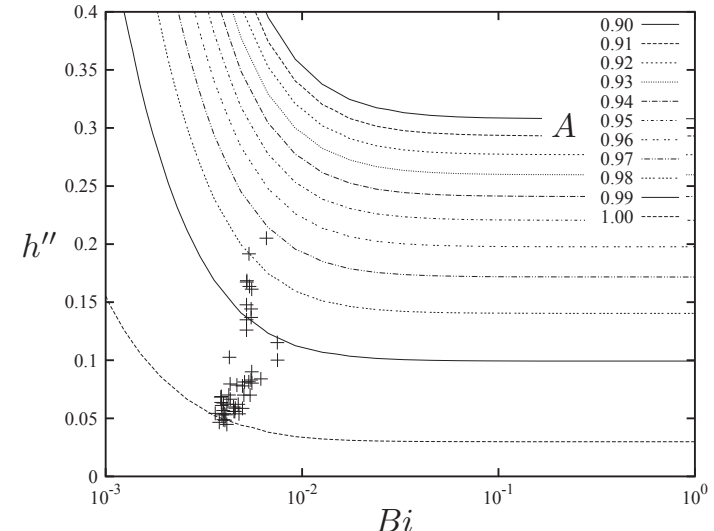

(b) $a_{1}^{*}=0.080$.

Figure 6: Augmentation contours as a function of $B i=\bar{h} a / k_{\mathrm{S}}$ and $h^{\prime \prime}=\sigma_{h} / \bar{h}$, with the positions of experimental data shown.

and reliable prediction of the time-averaged heat transfer in strongly unsteady flows with large variations in the HTC must consider the contribution of augmentation.

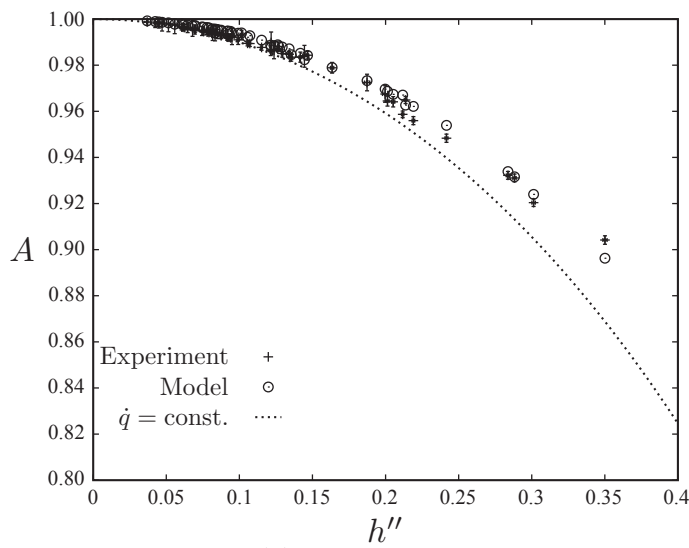

(a) $a_{1}^{*}=0.056$

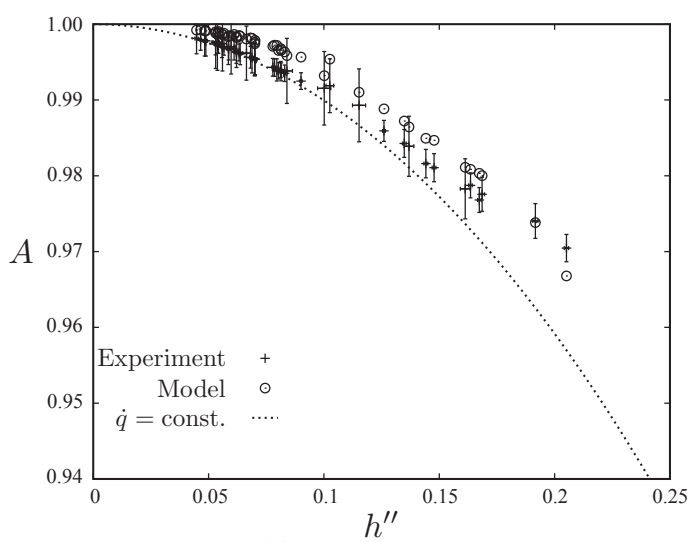

(b) $a_{1}^{*}=0.080$.

Figure 7: Measured augmentation effect against $h^{\prime \prime}$ including the corresponding modelled points.

Finally, as noted in Section 2.2, the heat flux fluctuations were relatively small, although still finite, at the measurement position, which in these experiments was chosen to be $\sim 300 d_{0}$ downstream of the inlet. In the long length limit, when the film flow becomes fully thermally developed, the inner wall heat flux fluctuations will tend to zero, and the inner wall heat flux a steady value. Consider this case, when the wall heat flux has become constant: $\dot{q}_{\mathrm{w}}^{\prime}=0, \dot{q}_{\mathrm{w}}=\bar{q}_{\mathrm{w}}$. In this case, the fluctuations in the wall-fluid temperature 
difference $\Delta T_{\mathrm{f}}$ are completely dependent on the HTC. The augmentation ratio $A$ then becomes,

$$
A=\frac{\overline{\dot{q}}_{\mathrm{w}}}{\bar{h} \overline{\Delta T_{\mathrm{f}}}}=\frac{\overline{\dot{q}}_{\mathrm{w}}}{\bar{h} \overline{\left(\dot{q}_{\mathrm{w}} / h\right)}}=\frac{1}{\bar{h} \overline{(1 / h)}}=\frac{1}{\int h P(h) d h \int P(h) / h d h} .
$$

Assuming a sinusoidal HTC, $h=\bar{h}+\widehat{h} \sin (\omega t)$, and using the binomial expansion of Eq. (15) gives,

$$
\frac{1}{A}=\overline{1-h^{*} \sin (\omega t)+h^{* 2} \sin ^{2}(\omega t)-\ldots},
$$

where $h^{*}=\widehat{h} / \bar{h}$ is the normalised HTC amplitude. By evaluating the mean over one cycle Eq. (16) becomes,

$$
\frac{1}{A}=1+\frac{h^{* 2}}{2}+\sum_{n} \frac{1}{2^{2 n}}\left(\begin{array}{c}
2 n \\
n
\end{array}\right) h^{* 2 n} ; \quad n=2 \ldots \infty
$$

The series in Eq. (17) will converge for $h^{*}<1$, and is shown as a function of $h^{\prime \prime}=h^{*} / \sqrt{2}$ in Fig. 7. This relation will be considered when the modelled and measured augmentation ratios are compared.

Retuning to Fig. 7, we can now compare the actual experimental results, and hence also the similar theoretical results from the full conjugate model, to the simplified model above which assumes $\dot{q}_{\mathrm{w}}^{\prime}=0$ thus leading to Eq. (17). A small difference can be identified between the measured values of $A$, and the corresponding values obtained for the limiting case described by Eq. (17). Specifically, this perfectly isoflux inner wall approximation always leads to a notable overprediction of the augmentation effect, or an underprediction of the value of $A$, even though the amplitude of the heat flux fluctuations $\dot{q}_{\mathrm{w}}^{\prime}$ on the inner wall was confirmed by the measurements to amount to only $0.6 \%$ of $\overline{\dot{q}}_{\mathrm{w}}$, as stated in Section 2.2. Recall that deviations of $A$ from unity signify a greater augmentation effect. Hence, one may conclude that, depending on the accuracy required, it may be necessary to solve the full conjugate problem for the prediction of the heat exchange characteristics, even in problems with limited conjugation. 


\section{Case Study II: Backwards-Facing Step}

Nakamura [16-18] has recently conducted detailed spatiotemporally resolved measurements of convective heat transfer in the flow behind a backwards-facing step. In this configuration a fluid, in this case air, flows over a long plate (with a trip-wire that induces transition to turbulence) and then over a backward (downward) step on the surface of the plate, positioned perpendicular to the flow direction. The developing turbulent boundary layer separates as air passes over the step and reattaches downstream of the step, resulting in a turbulent recirculating flow region behind the step, as illustrated in Fig. 8. The resulting flow has been studied extensively $[35,36]$ as a model problem for numerical simulations $[37,38]$ and experiments [39]. It is conventional in these problems to characterise the size of the turbulent recirculation region behind the step by the step height $H$, and to characterise the flows by a Reynolds number $R e$ based on the free-stream velocity $U_{\infty}$ and by the step height $H, R e_{H}=U_{\infty} H / \nu$, with $\nu_{\mathrm{f}}$ the kinematic viscosity of the fluid.

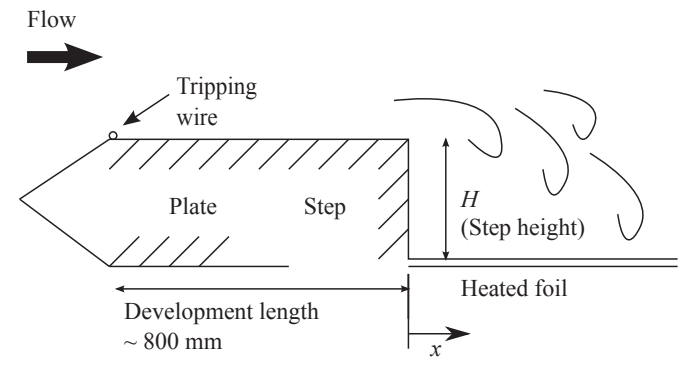

Figure 8: Schematic diagram of the backwards-facing step.

The heat transfer taking place behind such a step may be enhanced due to the increased mixing of the flow, which has obvious applications to heat exchange. Yet furthermore, the unsteadiness of the heat transfer process may result in temperature fluctuations on the surface of the wall. The amplitude of these fluctuations can be an important issue, for example, when avoiding thermal damage in a combustion chamber. In addition, the complex turbulent velocity field will be unsteady and gives rise to a time-varying HTC $h$. This is turn can lead to non-zero augmentation, and is the subject of the present study.

Direct numerical simulations (DNS) have been performed on these flows [37, 38] and have provided great insight into the turbulent flow field and its effect on the heat transfer behaviour. Nevertheless, these computations require great resources and long computational times for completion. In addition, each single simulation corresponding to only one design or operational point. Considerable effort and time needs to placed on spanning a range of variables and problem parameters, that can then allow a designer to extract and understand any underlying heat transfer trends. Using a simple model of the flow and heat transfer problem that includes explicitly the unsteadiness and conjugation that can arise, such as that proposed herein, can lead to a faster extraction of the important parameters and main trends, though of course there 
is the inevitable loss of some information relative to the DNS. By reference to suitable experimental data [1618], we will demonstrate the ability of our technique to model the behaviour of this unsteady heat transfer problem (specifically the augmentation ratio), and consequently to predict accurately the effects of changes to the solid material or thickness on the heat exchange characteristics.

In the experiments performed by Nakamura [16-18] the area behind the step was electrically heated by a thin ( $2 \mu \mathrm{m}$ thick) Titanium foil. The instantaneous foil temperature $T_{\mathrm{w}}$ was measured with an IR camera at high frequency and the instantaneous heat flux through the foil $\dot{q}_{\mathrm{w}}$ was calculated by evaluating an inverse heat transfer problem. From the knowledge of the free-stream $T_{\mathrm{f}, \infty}$ and instantaneous foil temperature $T_{\mathrm{w}}$, and the heat flux through the foil $\dot{q}_{\mathrm{w}}$, it was then possible to calculate the instantaneous HTC at any position on the foil surface, $h=\dot{q}_{\mathrm{w}} / \Delta T_{\mathrm{f}}$ with $\Delta T_{\mathrm{f}}=T_{\mathrm{f}, \infty}-T_{\mathrm{w}}$, as in Fig. $9(\mathrm{a})$.

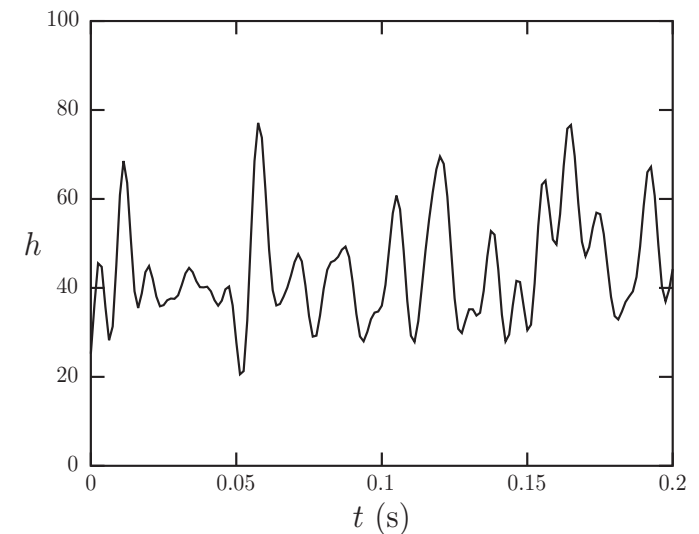

(a) Time-varying $\mathrm{HTC}$ at a point in the centre of the plate at $R e_{H}=4260$.

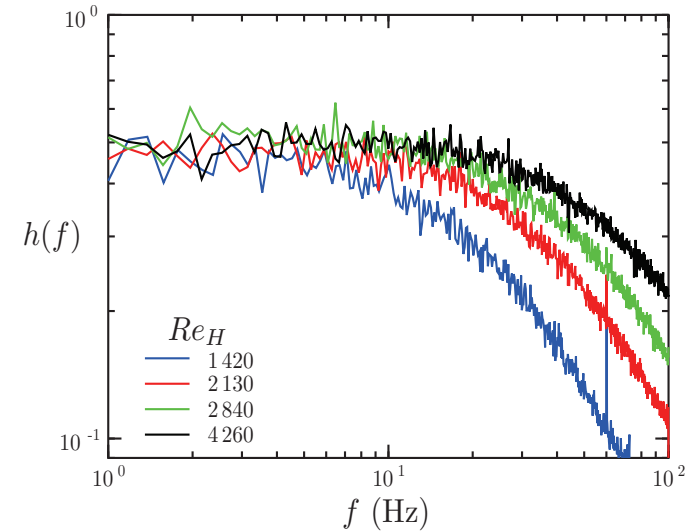

(b) Spatially averaged profile of the frequency content of $h$ over the foil at different $R e_{H}$.

$(\mathrm{Hz})$

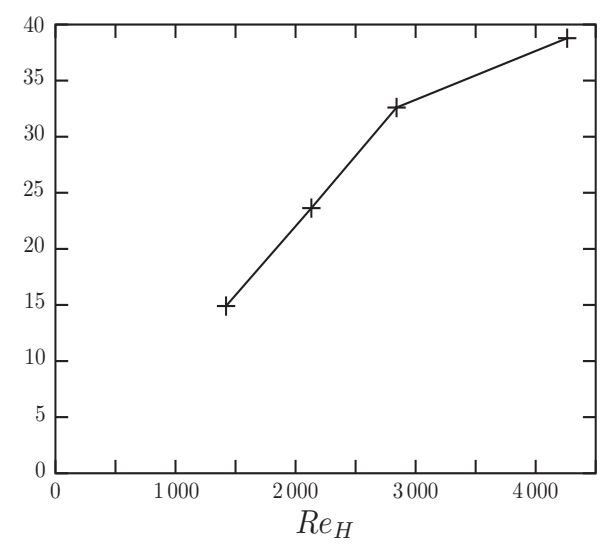

(c) Roll-off frequency of the HTC at different $R e_{H}$.

Figure 9: Experimentally measured variations in the HTC. 


\subsection{Modelling Procedure}

Our modelling approach requires the heat capacity ratio $C_{\mathrm{R}}=\left(\rho_{\mathrm{f}} c_{\mathrm{f}} / \rho_{\mathrm{s}} c_{\mathrm{s}}\right)(d / a)$ between the air flow and the Titanium foil to be calculated, as well as the Biot number $B i$ and the dimensionless solid thickness $a_{n}^{*}=a / \delta_{\mathrm{s}, n}$, which is the thickness of the solid $a(=2 \mu \mathrm{m})$ divided by the characteristic lengthscale of the fluctuations penetration into the solid $\delta_{\mathrm{s}, n}$. The fluid domain extent $d$ is chosen to be the thickness of the thermal boundary layer. The height of the turbulent recirculation region is known to be $3 \mathrm{~mm}$ greater than the step height $H(=10 \mathrm{~mm})$, from Ref. [17]. Scaling this by the Prandtl number for air, for which a value of $\operatorname{Pr}=0.71$ is used, gives an approximation for the thickness of the thermal boundary layer behind the step of $d=9.3 \mathrm{~mm}$. The data in Table 2 can then be used to evaluate $C_{\mathrm{R}}$, which for this configuration is found to be equal to about 2.3 , and the Biot number $B i=\bar{h} a / k_{\mathrm{s}}$, which is found to be between $1.9 \times 10^{-6}$ and $4.2 \times 10^{-6}$ depending on the value of $\bar{h}$, which is itself a function of position, but also of the flow $R e_{H}$. As expected, the Biot number $B i$ is small, due to the small thickness of the heated foil $a$.

Table 2: Thermal properties of materials in the experiments by Nakamura $[16,17]$. The thermal properties of air are at the film temperature, i.e. the mean temperature between the wall and the free stream.

\begin{tabular}{lccc}
\hline & $\begin{array}{c}\text { Density, } \rho \\
\left(\mathrm{kg} \mathrm{m}^{-3}\right)\end{array}$ & $\begin{array}{c}\text { Heat capacity, } c \\
\left(\mathrm{~J} \mathrm{~kg}^{-1} \mathrm{~K}^{-1}\right)\end{array}$ & $\begin{array}{c}\text { Thermal conductivity, } k \\
\left(\mathrm{~W} \mathrm{~m}^{-1} \mathrm{~K}^{-1}\right)\end{array}$ \\
\hline Air & 1.18 & 1005 & 0.026 \\
Titanium & 4510 & 540 & 21.9 \\
\hline
\end{tabular}

Returning to the frequency content of $h$ in Fig. 9(b), it is observed that the signal is largely broadband in nature. The spectra do not show any dominant peaks, only a slow first-order roll-off above a certain frequency that changes with each flow condition, i.e. $R e_{H}$. The values of the 3 -dB bandwidth frequency $f_{\mathrm{c}}$ (or, halfpower point) related to the first-order roll-off (defined as the frequency at which the content reaches $70 \%$ of its peak) for the four flows in Fig. 9(b) are plotted in Fig. 9(c). It can be seen that $f_{\mathrm{c}}$ increases monotonically at progressively higher $R e_{H}$. The broadband nature of the signals also leads to a range of thermal diffusion lengths over which unsteady heat transfer occurs, since $\delta_{\mathrm{s}, n}=\sqrt{2 \alpha_{\mathrm{s}} / \omega_{n}}$. After substituting the physical property values from Table 2 for the Titanium foil, an expression for the dimensionless foil thickness as a function of frequency can be obtained as, $a^{*}(\omega)=a / \delta_{\mathrm{s}}(\omega)=4.7 \times 10^{-4} \sqrt{\omega}$. Now, the largest 3-dB roll-off frequency $f_{\mathrm{c}}$ of the broadband thermal responses from the experimental data was $\sim 40 \mathrm{~Hz}$, from Fig. 9(c). Based on this value of $f_{\mathrm{c}}$, the largest dimensionless solid thickness $a^{*}$ observed in all of the experiments was about $7.4 \times 10^{-3}$. Furthermore, the rate at with the experimental data was sampled results in a Nyquist frequency of $200 \mathrm{~Hz}$, which is the highest resolvable frequency in the signals. By means of comparison, this corresponds to a dimensionless solid thickness $a^{*}$ of $1.7 \times 10^{-2}$. These small values of $a^{*}(\ll 1)$ would suggest that the solid surface in contact with the thermal boundary layer (inner wall) will be strongly coupled to the back/underside surface of the foil that is facing away from the air flow (outer wall) and on which a constant 
heat flux boundary condition is imposed. Thus, it is unlikely to observe a high degree of conjugation in this experiment, although this is likely to be the case for thicker foils ${ }^{4}$.

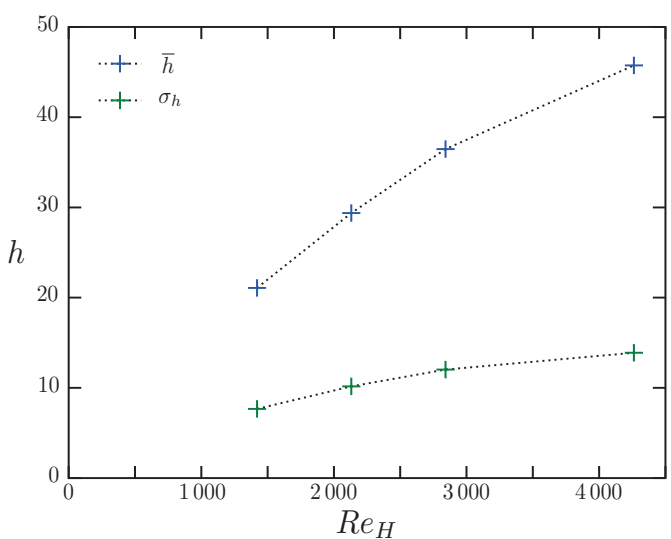

(a) Mean and standard deviation of $h$ over the foil for different $R e_{H}$.

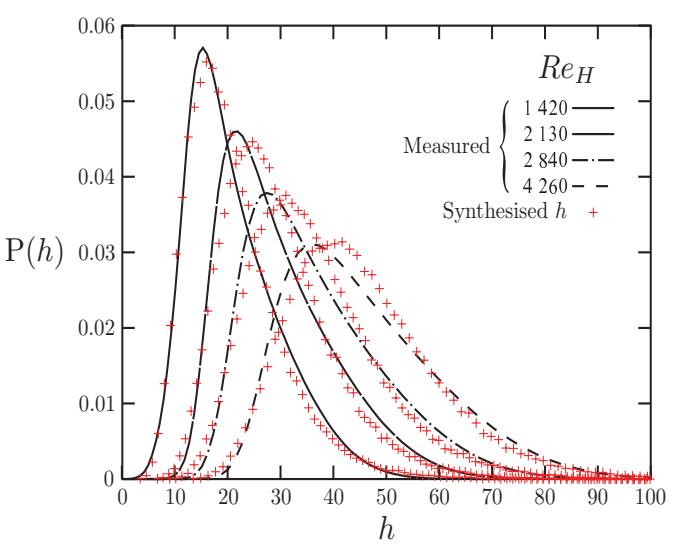

(b) Probability density of the experimental $h$ and a fitted lognormal distribution.

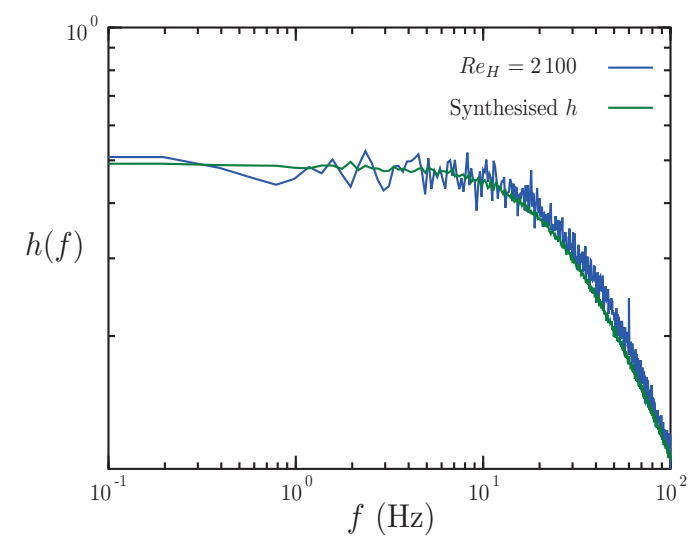

(c) A comparison of the frequency content of a synthesised $h$ to that of the corresponding experimental HTC at $R e_{H}=2100$.

Figure 10: Matching the frequency content and probability distribution of the measured and synthesised HTC. The frequency content of $h$ at $R e_{H}=2100$ is spatially averaged over the foil.

Our model was initially developed for sinusoidal time-variations at a single frequency, but for this problem it is necessary and relatively straightforward to modify the algorithm so as to accommodate arbitrary profiles of $h$ through a linear Fourier decomposition. The first step in this direction requires the generation of a synthesised $h$, which has a similar frequency content and a similar probability distribution to that measured

\footnotetext{
${ }^{4}$ It will be the case that a thicker foil should result in a decrease in the temperature fluctuation on the foil surface, approaching a steady wall temperature condition, however, that the same time it will results in an increase in the heat flux fluctuation as the solid becomes more decoupled from the boundary condition on the outer wall of the foil. Recall from [1] that our definition of a 'conjugate' problem involves both an unspecified time-varying temperature and heat flux at the common fluid-solid interface.
} 
in the experiments. Although the measured HTC signals can be used directly, an important aim here is not to devise a modelling strategy that depends on the provision of this highly detailed information. Probability densities of the measured $h$ are shown in Fig. 10(b) for the 4 flows with different $R e_{H}$, together with lognormal distributions based on the corresponding means and variances provided by the experimental data and shown separately in Fig. 10(a)). Both the mean $\bar{h}$ and standard deviation $\sigma_{h}$ of the HTC increase monotonically at higher $R e_{H}$. Further, the lognormal approximations resemble closely the directly measured probability densities of $h$. At this point it is noted that the frequency content of a normal distribution is flat, so this must be low-pass filtered in order to exhibit a roll-off similar to that in the measured HTCs in Fig. 9(b). An example of the spectral density of a synthesised $h$ after this filtering procedure has been performed is shown in Fig. 10(c). As previously stated, the cut-off frequencies were taken from the measured $h$ spectra (i.e. from Fig. 9(b)) and are plotted in Fig. 9(c) for the four different flow conditions.

The method employed for synthesising the HTC is computationally simple. Firstly, a sufficiently large number of randomly distributed samples are generated, using a lognormal distribution with the same statistical parameters seen in the experimental data (Fig. 10(a)). These samples are then low-pass filtered with the corresponding roll-off frequency seen in Fig. 9(c). Having obtained this synthesised $h$ it is possible to calculate the resulting temperature and heat flux response of the conjugate fluid-solid system for a given mean heat flux, as explained previously for Case V [1]. The statistics of the heat transfer behaviour can then be generated from this modelled response, such as the variation in temperature or the augmentation ratio A. Finally, since the HTC was generated randomly, a number of repeated trials are run in order to smooth out any random bias that may have been generated by the procedure for the synthesis of $h$.

\subsection{Model Predictions and Comparison to Experiment}

Comparing the spatially averaged measurement of augmentation loss over the measurement area, $A_{\text {loss }}=$ $1-A$, directly from the experimental data (Fig. 11(a)), with the stochastic prediction from our 1-D unsteady conjugate model employing some basic statistical information on the expected HTC as described in Section 3.1 (Fig. 11(b)), it can be seen that the model results exhibit a behaviour that is reasonably close to the observed data. The main conclusion concerning the augmentation in this flow scenario is that this is small and becomes progressively smaller at higher $R e_{H}$. This trend as well as the magnitude of the augmentation are captured well. And this, even though the model uses a relatively simple description for convective heat transfer based on Newton's law, and as such contains only superficial information on the underlying processes in the fluid. It can be concluded that if the distribution of the frequency content of $h$ is known to some approximation, for which the mean and variance of $h$ for a certain flow condition are required, the effect of the solid wall on the conjugate heat transfer can be modelled quite accurately. 


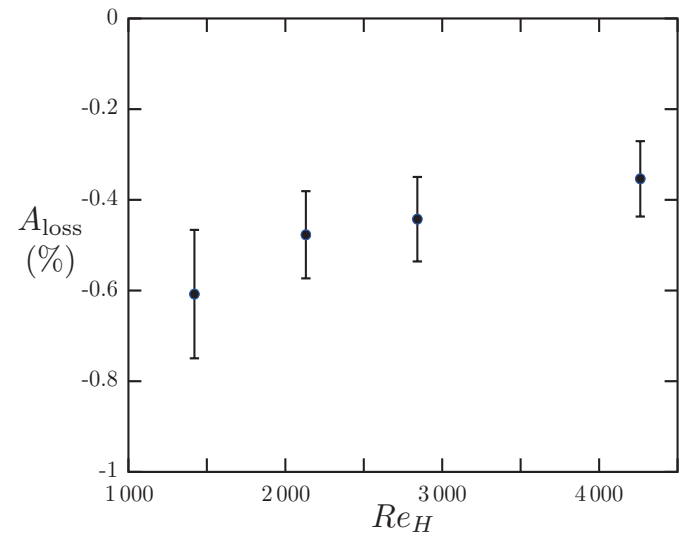

(a) Spatially averaged augmentation loss $1-A$ of over the foil for the four $\left(R e_{H}\right)$ flow cases.

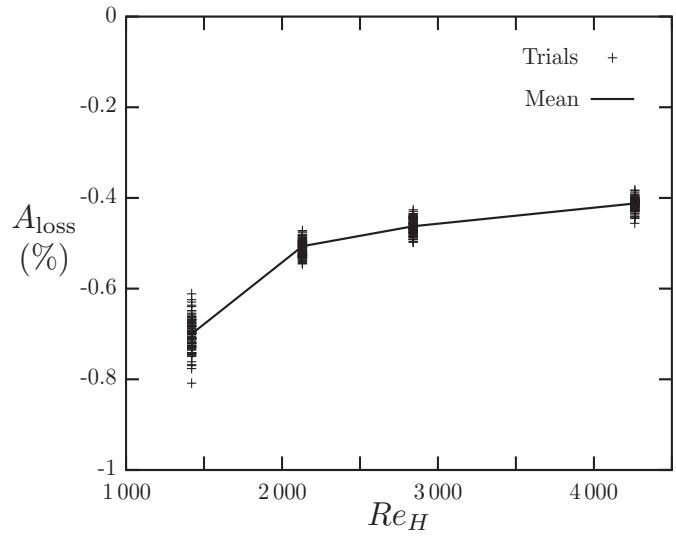

(b) Modelled augmentation loss $1-A$ from a synthesised $h$.

Figure 11: Augmentation loss $A_{\text {loss }}=1-A=-\overline{h^{\prime} \Delta T_{\mathrm{f}}^{\prime}} / \bar{h} \overline{\Delta T_{\mathrm{f}}}$ comparison: (a) from experimental data, and (b) modelled based on a synthesised $h$. The error bars represent, in: (a) the standard deviation of the variation in the augmentation loss over the foil, and in (b) results from different trial runs from which the average result is obtained.

There are many thermal-fluid applications where measurements of the turbulence or unsteady flow behaviour have been made. Using the technique presented here it may be possible to predict the effect of unsteadiness on the heat transfer performance (also, if relevant, in the conjugate case), and to calculate quantities unique to these problems such as the augmentation ratio. From this, the effects of simple geometrical modifications (i.e. thickness) and of the material properties of the thermal field in the solid and fluid can be better understood. In this particular demonstration the magnitude of augmentation is relatively small. Yet, the model reveals that in applications with a smaller dimensionless foil thickness $a^{*}$ (e.g. in unsteady flows with slower frequencies), a larger Biot number $B i$ (e.g. a larger mean $h$ ), a smaller heat capacity ratio $C_{\mathrm{R}}$, and a larger fluctuation in $h$, this effect would become much more significant. 


\section{Conclusions}

A simple semi-analytical 1-D model of conjugate heat transfer with a time-varying heat transfer coefficient has been applied to two different non-isothermal/diabatic problems, a heated falling planar fluid film, and the separated and re-attached flow of a turbulent boundary layer behind a backwards-facing step. The model predictions of the augmentation effects in these problems were then compared to actual experimental results from each case. Such simple and efficient models allow for an understanding of the dominant underlying mechanisms of the heat transfer problem, and allow the effects of a wide range of materials selection and geometry parameters to be evaluated quickly and reliably for engineering design.

The first problem considered was that of a fluid film flowing over a heated flat plate, with (excited) waves resulting in a fluctuation in the heat transfer coefficient. The heat transfer coefficient was found to have an inverse relationship with film thickness as expected from Nusselt theory. However, the measured Nusselt number was slightly higher than that predicted by the idealised Nusselt flow (i.e. fully developed, completely steady and laminar flow). It was found that the heat flux fluctuation at the measurement location was low, but finite. This resulted due to the coupling of the fluid temperature to the fluctuations in the heat transfer coefficient as the fluid convected with (and at the speed as) the fluctuations in the heat transfer caused by the waves. A good agreement was found between the measured and predicted augmentation ratio. The augmentation ratio was always negative, resulting in a reduction in heat transfer with respect to the steady case of up to $10 \%$. At the range of values of Biot number and dimensionless solid thickness that the experiment was performed, it was observed that the augmentation ratio depended on both the amplitude of the heat transfer coefficient and, to a lesser extend, the Biot number. Under the assumption of a perfectly isoflux inner wall condition, an augmentation ratio for a given heat transfer coefficient was also calculated. However, this assumption consistently overpredicted the augmentation effect, despite the fact that inner wall heat flux fluctuations were confirmed by the measurements as being small. It can be inferred that the model correctly accounts for fluctuations in fluid or solid temperatures, and the heat transfer coefficient.

The model was then extended to investigate broadband heat transfer coefficients and results were compared to high-resolution experimental data provided for a backwards-facing step. Using simple statistical information on the heat transfer coefficient from the measurements in this flow (the mean, variance and spectral frequency cut-off), the model correctly predicted the observed trends and the approximate magnitude of augmentation exhibited in this experimental application over a range of Reynolds numbers.

Generally, very good agreement was found between the experimental and predicted augmentation ratios. The augmentation effect was negative in both considered flow cases, with the region of greatest (negative) augmentation occurring at high values of the Biot number and high heat transfer coefficient fluctuation intensities in the film flows, and at low Reynolds numbers in the backwards-facing step flows. 


\section{Acknowledgements}

RM would like to acknowledge the DTA studentship awarded to him by the Department of Chemical Engineering, Imperial College London, and EPSRC without which he would not have been able to conduct this research. We would also like to acknowledge the use made of the FLIR SC3000 IR imager, and Cedip

Titanium IR imager, which was borrowed from the EPSRC (Engineering and Physical Sciences Research Council) Instrument Pool. 


\section{References}

[1] R. Mathie, C.N. Markides, Augmentation in unsteady conjugate heat transfer Part I: Semi-analytical 1-D framework, International Journal of Heat and Mass Transfer, submitted, in peer review

[2] W.M. Kays, A.L. London, Compact heat exchangers, $3^{\text {rd }}$ ed., Krieger Publishing Company, 1998. ISBN:9781575240602

[3] P. Bouvier, P. Stouffs, J.-P. Bardon, Experimental study of heat transfer in oscillating flow, International Journal of Heat and Mass Transfer 48 (12) (2005) 2473-2482. doi:10.1016/j.ijheatmasstransfer.2005.01.037

[4] T.S. Zhao, P. Cheng, Oscillatory heat transfer in a pipe subjected to a periodically reciprocating flow, Journal of Heat Transfer 118 (3) (1996) 592-597. doi:10.1115/1.2822673

[5] A. Sorin, F. Bouloc, B. Bourouga, P. Anthoine, Experimental study of periodic heat transfer coefficient in the entrance zone of an exhaust pipe, International Journal of Thermal Sciences 47 (12) (2008) 1665-1675. doi:10.1016/j.ijthermalsci.2008.01.006

[6] K.C. Leong, L.W. Jin, An experimental study of heat transfer in oscillating flow through a channel filled with an aluminum foam, International Journal of Heat and Mass Transfer 48 (2) (2005) 243-253. doi:10.1016/j.ijheatmasstransfer.2004.08.025

[7] J.-C. Yu, Z.-X. Li, T.S. Zhao, An analytical study of pulsating laminar heat convection in a circular tube with constant heat flux, International Journal of Heat and Mass Transfer 47 (24) (2004) 5297-5301. doi:10.1016/j.ijheatmasstransfer.2004.06.029

[8] M.A. Habib, A.M. Attya, A.I. Eid, A.Z. Aly, Convective heat transfer characteristics of laminar pulsating pipe air flow, Heat and Mass Transfer 38 (3) (2002) 221-232. doi:10.1007/s002310100206

[9] M. Faghri, K. Javdani, A. Faghri, Heat transfer with laminar pulsating flow in a pipe, Letters in Heat and Mass Transfer 6 (4) (1979) 259-270. doi:10.1016/0094-4548(79)90013-4

[10] M.R. Mackley, P. Stonestreet, Heat transfer and associated energy dissipation for oscillatory flow in baffled tubes, Chemical Engineering Science 50 (14) (1995) 2211-2224. doi:10.1016/0009-2509(95)00088$\mathrm{M}$

[11] H.N. Hemida, M.N. Sabry, A. Abdel-Rahim, H. Mansour, Theoretical analysis of heat transfer in laminar pulsating flow, International Journal of Heat and Mass Transfer 45 (8) (2002) 1767-1780. doi:10.1016/S0017-9310(01)00274-5 
[12] R. Siegel, M. Perlmutter, Heat transfer for pulsating laminar duct flow, Journal of Heat Transfer 84 (1962) 111-123. doi:10.1115/1.3684307

[13] H. Chattopadhyay, F. Durst, S. Ray, Analysis of heat transfer in simultaneously developing pulsating laminar flow in a pipe with constant wall temperature, International Communications in Heat and Mass Transfer 33 (4) (2006) 475-481. doi:10.1016/j.icheatmasstransfer.2005.12.008

[14] H.W. Cho, J.M. Hyun, Numerical solutions of pulsating flow and heat transfer characteristics in a pipe, International Journal of Heat and Fluid Flow 11 (4) (1990) 321-330. doi:10.1016/0142-727X(90)90056-H

[15] S.Y. Kim, B.H. Kang, J.M. Hyun, Heat transfer in the thermally developing region of a pulsating channel flow, International Journal of Heat and Mass Transfer 36 (17) (1993) 4257-4266. doi:10.1016/00179310(93)90088-N

[16] H. Nakamura, Spatio-temporal measurements of convective heat transfer for a backward-facing step flow (Paper YN-024), in: Proceedings of the $6^{\text {th }}$ International Symposium on Turbulence Heat and Transfer (THMT '09), Sapienza Università di Roma, Rome, Italy, 2009, pp. 1-12

[17] H. Nakamura, Spatio-temporal measurement of convective heat transfer for the separated and reattaching flow (Paper IHTC14-22753), in: Proceedings of the $14^{\text {th }}$ International Heat Transfer Conference (IHTC14), Washington, WA, 2010, pp. 1-9

[18] H. Nakamura, Frequency response and spatial resolution of a thin foil for heat transfer measurements using infrared thermography, International Journal of Heat and Mass Transfer 52 (21-22) (2009) 50405045. doi:10.1016/j.ijheatmasstransfer.2009.04.019

[19] V.V. Lel, F. Al-Sibai, A. Leefken, U. Renz, Local thickness and wave velocity measurement of wavy films with a chromatic confocal imaging method and a fluorescence intensity technique, Experiments in Fluids 39 (5) (2005) 856-864. doi:10.1007/s00348-005-0020-x

[20] K. Moran, J. Inumaru, M. Kawaji, Instantaneous hydrodynamics of a laminar wavy liquid film, International Journal of Multiphase Flow 28 (5) (2002) 731-755. doi:10.1016/S0301-9322(02)00006-X

[21] W. Nusselt, Die oberflächenkondensation des wasserdampfes, Zeitschrift des Vereines Deutscher Ingenieure 60 (4) (1916) 541-546, 569-575

[22] O.A. Kabov, E.A. Chinnov, J.-C. Legros, Three-dimensional deformations in non-uniformly heated falling liquid film at small and moderate Reynolds numbers, in: Proceedings of the $2^{\text {nd }}$ International Berlin Workshop on Transport Phenomena with Moving Boundaries (IBW2), Berlin, Germany, 2004, pp. $62-80$ 
[23] O.A. Kabov, B. Scheid, I.A. Sharina, J.-C. Legros, Heat transfer and rivulet structures formation in a falling thin liquid film locally heated, International Journal of Thermal Science 41 (7) (2002) 664-672. doi:10.1016/S1290-0729(02)01361-3

[24] B. Scheid, S. Kalliadasis, C. Ruyer-Quil, P. Colinet, Interaction of three-dimensional hydrodynamic and thermocapillary instabilities in film flows, Physical Review E 78 (6) (2008) 066311. doi:10.1103/PhysRevE.78.066311

[25] A. Criminisi, I. Reid, A. Zisserman, A plane measuring device, Image and Vision Computing 17 (8) (1999) 625-634.

[26] P. Chamarthy, S.V. Garimella, S.T. Wereley, Measurement of the temperature non-uniformity in a microchannel heat sink using microscale laser-induced fluorescence, International Journal of Heat and Mass Transfer 53 (15-16) (2010) 3275-3283. doi:10.1016/j.ijheatmasstransfer.2010.02.052

[27] L. Shi, X. Mao, A.J. Jaworski, Application of planar laser-induced fluorescence measurement techniques to study heat transfer characteristics of parallel-plate heat exchangers in thermoacoustic devices, Measurement Science and Technology 21 (11) (2010) 115405 1-16. doi:10.1088/0957-0233/21/11/115405

[28] L. Shi, Z. Yu, A.J. Jaworski, Application of laser-based instrumentation for measurement of timeresolved temperature and velocity fields in the thermoacoustic system, International Journal of Thermal Sciences 49 (9) (2010) 1688-1701. doi:10.1016/j.ijthermalsci.2010.03.015

[29] D. Ross, M. Gaitan, L.E. Locascio, Temperature measurement in microfluidic systems using a temperature-dependent fluorescent dye, Analytic Chemistry 73 (17) (2001) 4117-4123. doi:10.1021/ac0103701

[30] A. Schagen, M. Modigell, G. Dietze, R. Kneer, Simultaneous measurement of local film thickness and temperature distribution in wavy liquid films using a luminescence technique, International Journal of Heat and Mass Transfer 49 (25-26) (2006) 5049-5061. doi:10.1016/j.ijheatmasstransfer.2006.06.010

[31] A. Schagen, M. Modigell, Local film thickness and temperature distribution measurement in wavy liquid films with a laser-induced luminescence technique, Experiments in Fluids 43 (2-3) (2007) 209221. doi:10.1007/s00348-007-0289-Z

[32] V.V. Lel, A. Kellermann, G. Dietze, R. Kneer, A.N. Pavlenko, Investigations of the Marangoni effect on the regular structures in heated wavy liquid films, Experiments in Fluids 44 (2) (2008) 341-354. doi:10.1007/s00348-007-0408-x 
[33] F. Zhang, X. Zhao, J. Geng, Y.-T. Wu, Z. Zhang, A new insight into Marangoni effect in nonisothermal falling liquid films, Experimental Thermal and Fluid Science 31 (4) (2007) 361-365. doi:10.1016/j.expthermflusci.2006.05.008

[34] F. Zhang, Y.-T. Wu, J. Geng, Z.-B. Zhang, An investigation of falling liquid films on a vertical heated/cooled plate, International Journal of Multiphase Flow 34 (1) (2008) 13-28. doi:10.1016/j.ijmultiphaseflow.2007.08.003

[35] D.W. Etheridge, P.H. Kemp, Measurements of turbulent flow downstream of a rearward-facing step, Journal of Fluid Mechanics 86 (3) (1978) 545-566. doi:10.1017/S0022112078001275

[36] E.V. Shishov, P.S. Roganov, S.I. Grabarnik, V.P. Zabolotsky, Heat transfer in the recirculating region formed by a backward-facing step, International Journal of Heat and Mass Transfer 31 (8) (1988) 15571562. doi:10.1016/0017-9310(88)90267-0

[37] R.V.R. Avancha, R.H. Pletcher, Large eddy simulation of the turbulent flow past a backward-facing step with heat transfer and property variations, International Journal of Heat and Fluid Flow 23 (5) (2002) 601-614. doi:10.1016/S0142-727X(02)00156-X

[38] P.R. Kanna, M.K. Das, Conjugate heat transfer study of backward-facing step flow - A benchmark problem, International Journal of Heat and Mass Transfer 49 (21-22) (2006) 3929-3941. doi:10.1016/j.ijheatmasstransfer.2006.02.058

[39] S. Terhaar, A. Velazquez, J.R. Arias, M. Sanchez-Sanz, Experimental study on the unsteady laminar heat transfer downstream of a backwards facing step, International Communications in Heat and Mass Transfer 37 (5) (2010) 457-462. doi:10.1016/j.icheatmasstransfer.2010.01.009 


\section{Appendix A. 1-D Solid Thermal Response}

Thermal conduction in a homogeneous solid across a finite 1-D domain is a classic example of an Initial Bound Value Problem (IBVP). The heat flux conducted through the solid is described by Fourier's law, $\dot{q}(y, t)=-k_{\mathrm{s}} \partial T(y, t) / \partial y$. In the problem considered here the thermal conductivity $k_{\mathrm{s}}$, which is a property of the solid material, is isotropic across the spatial domain and is independent of time and temperature. Fourier's law can be combined with the energy equation in the solid domain to produce a partial differential equation (PDE) describing the evolution of temperature across the solid,

$$
\frac{\partial T(y, t)}{\partial t}=\alpha_{\mathrm{s}} \frac{\partial^{2} T(y, t)}{\partial y^{2}},
$$

where $\alpha_{\mathrm{s}}$ is the thermal diffusivity of the solid. Taking the Laplace transform $\mathcal{L}\{f(t)\}=\int_{0^{-}}^{\infty} e^{-s t} f(t) d t$ of the PDE in Eq. (A.1) gives a representation of the IBVP in the Laplace domain,

$$
s \hat{T}(y, s)-T(y, 0)=\alpha_{\mathrm{s}} \frac{\partial^{2} \hat{T}(y, s)}{\partial y^{2}} .
$$

\section{A.1. General Solution}

For the case when the initial temperature across the solid is uniform, $T(y, 0)=0$, the Laplace representation of the IBVP problem in Eq. (A.2) becomes a linear homogeneous ordinary differential equation (ODE) of the Laplace representation of the temperature $\hat{T}$ across the spatial domain $y$. Substituting a generic exponential function of the form $\hat{T}(y, s)=K e^{\lambda y}$ into Eq. (A.2) results in a relation for the coefficients of the exponential,

$$
\lambda= \pm p ; p=\sqrt{\frac{s}{\alpha_{\mathrm{s}}}} .
$$

Here, $s$ is the Laplace variable in the complex $s$ domain and $p=\sqrt{s / \alpha_{\mathrm{s}}}$ can be seen as a Laplace variable in the $p$ domain. Thus, the complimentary function of the temperature profile solution in the solid is,

$$
\hat{T}(y, s)=A e^{p y}+B e^{-p y} .
$$

and the use of Fourier's law together with Eq. (A.4) gives the heat flux distribution through the solid,

$$
\hat{q}(y, s)=-k_{\mathrm{s}} p\left(A e^{p y}-B e^{-p y}\right),
$$

For an isoflux condition imposed on the 'outer' surface, $\hat{q}(y=0, s)=0$, the coefficients must take the form $A=B$, whereas for an isothermal condition on the same boundary, $\hat{T}(0, s)=0$, they must obey $A=-B$.

\section{A.2. Transfer Functions}

The temperature distribution within the solid is usually of limited interest in typical heat transfer problems. Of greater importance are the temperatures and heat fluxes at the boundaries of a finite solid domain. These 
